\begin{tabular}{|c|c|c|c|c|c|c|}
\hline \multirow{3}{*}{$\begin{array}{l}\text { 2. ECN Category } \\
\text { (mark one) } \\
\\
\text { Supplemental } \\
\text { Direct Revision } \\
\text { Change ECN } \\
\text { Temporary } \\
\text { Standby } \\
\text { Supersedure } \\
\text { Cancel/Noid } \\
\end{array}$} & \multirow{3}{*}{$\begin{array}{r}0 \\
{[x]} \\
{[]} \\
{[]} \\
{[]} \\
{[]} \\
{[]}\end{array}$} & \multicolumn{2}{|c|}{$\begin{array}{l}\text { 3. Originator's Name, Organization, MSIN, } \\
\text { and Telephone No. } \\
\text { J.S. Hammers/TRS/R3-25/372-2657 }\end{array}$} & \multicolumn{2}{|c|}{$\begin{array}{l}\text { 4. USO Required? } \\
5 \times 8 \geqslant / 2 / 99 \\
\text { txtyes N No }\end{array}$} & $\begin{array}{l}\text { 5. Date } \\
6 / 2 / 99\end{array}$ \\
\hline & & \multicolumn{2}{|c|}{$\begin{array}{l}\text { 6. Project Title/No. Work Order No. } \\
\qquad \begin{array}{l}\text { W-314, Tank Farm Restoration and Safe } \\
\text { Operations/ W-314 }\end{array}\end{array}$} & \multicolumn{2}{|c|}{ 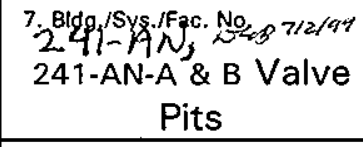 } & $\begin{array}{l}\text { 8. Approval Designator } \\
\text { ES Q } \\
\cos 112199\end{array}$ \\
\hline & & \multicolumn{2}{|c|}{$\begin{array}{l}\text { 9. Document Numbers Changed by this ECN } \\
\text { (includes sheet no. and rev.) } \\
\qquad \begin{array}{l}\text { HNF-1733, Rev 3, } \\
\text { HNF-1902, Rev 3, }\end{array}\end{array}$} & \multicolumn{2}{|c|}{$\begin{array}{l}\text { 10. Related ECN No(s). } \\
\qquad \text { N/A }\end{array}$} & $\begin{array}{l}\text { 11. Related PO No. } \\
\text { NA }\end{array}$ \\
\hline 12a. Modification Work & & $\begin{array}{l}\text { 12b. Work Package } \\
\text { No. }\end{array}$ & \multicolumn{2}{|c|}{ 12c. Modification Work Complete } & \multicolumn{2}{|c|}{$\begin{array}{l}\text { 12d. Restored to Original Condi- } \\
\text { tion (Temp. or Standby ECN only) }\end{array}$} \\
\hline $\begin{array}{l}\text { [] Yes (fill out Blk. } \\
\text { (2b) }\end{array}$ & & NA & \multicolumn{2}{|l|}{ NA } & \multicolumn{2}{|r|}{ NA } \\
\hline $\begin{array}{c}{[x] \text { No (NA BIks. 12b, }} \\
12 c, 12 d\}\end{array}$ & & & \multicolumn{2}{|c|}{$\begin{array}{c}\text { Design Authority/Cog. Engineer } \\
\text { Signature \& Date } \\
\end{array}$} & \multicolumn{2}{|c|}{$\begin{array}{c}\text { Design Authority/Cog. Engineer } \\
\text { Signature \& Date }\end{array}$} \\
\hline
\end{tabular}

13a. Description of Change

13b. Design Baseline Document? [X] Yes [] No

241-AN-A and 241-AN-B Valve Pit Specific Test \& Evaluation Plan, HNF-1733, Rev 4 and HNF-1902, Rev 4. Revised construction testing to reflect current project scope. Digrar 84 $7 / 12 / 99$

14a. Justification (mark one)

Criteria Change []

As-Found

[] Design Improvement

[x]

Environmental

[]

Facility Deactivation

[]

14b. Justification Details

Corrected W-314 241-AN-A and 241-AN-B Valve Pit Specific Test \& Evaluation Plans to reflect current Project Status.

USQ is not reguived per ANF-IP. $084200 L \bar{L}$ Section 5.4 , reU ilb. The STEPs inand of themselves are notwork authory zingand dono make any phy sicarchanges to the taulc farm faclities. areno

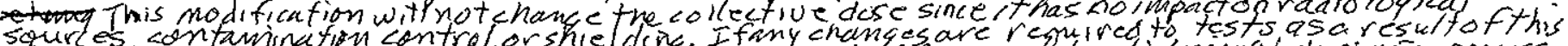

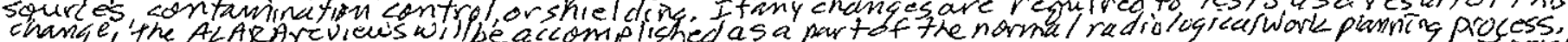

15. Distribution (include name, MSIN, and no. of copies) RELEASE STAMP

See attached Distribution Sheet, page 3. 


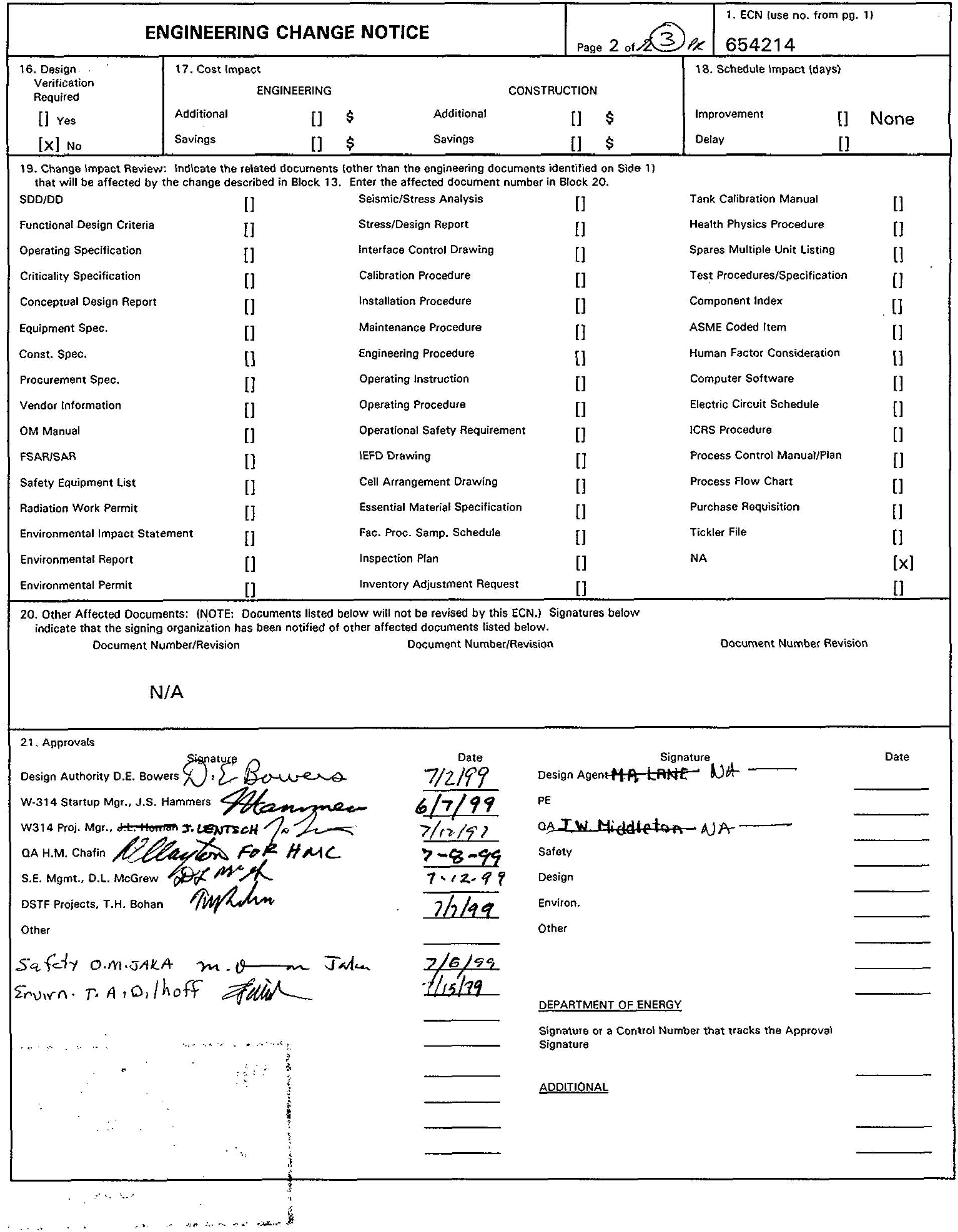




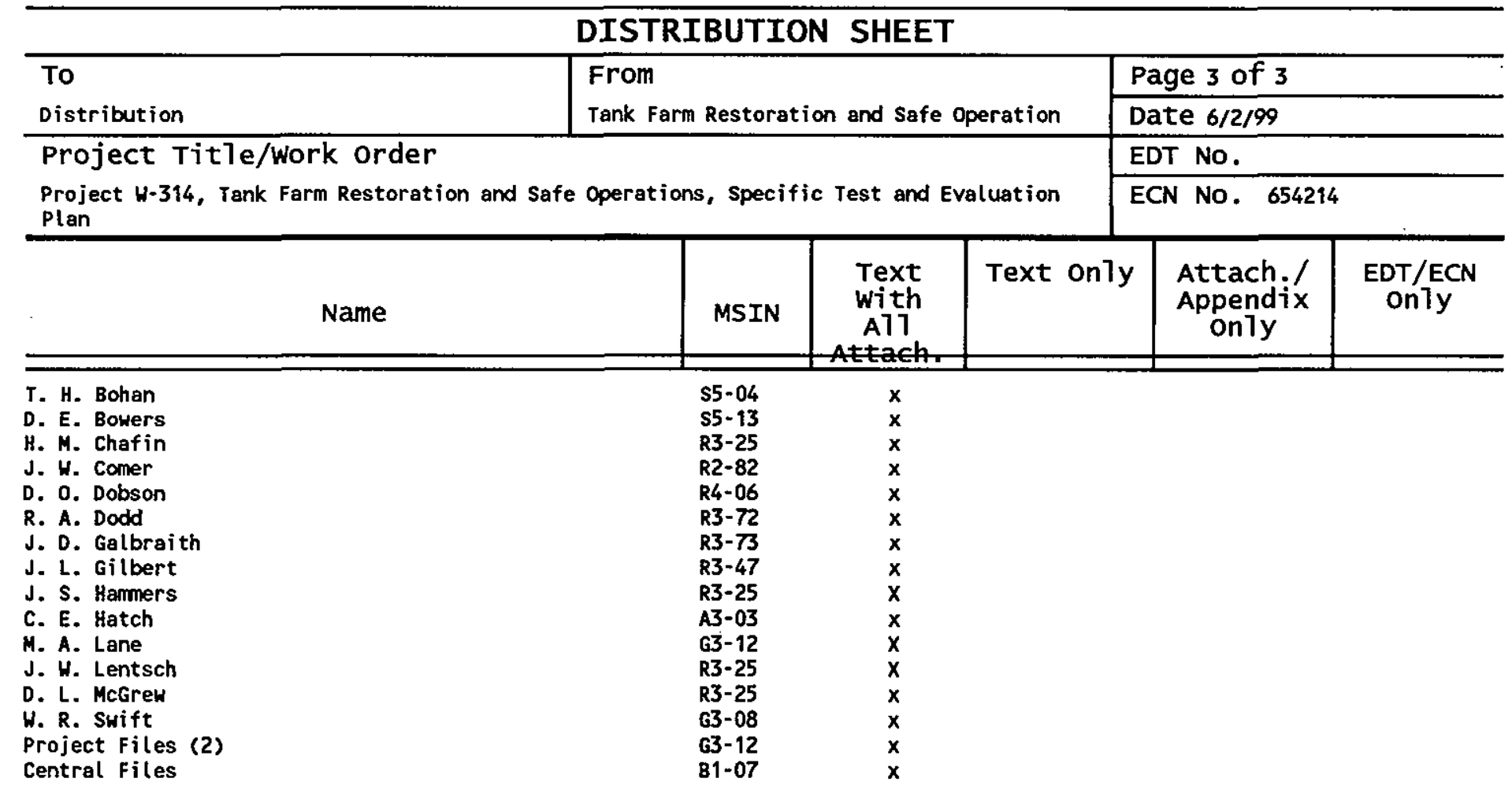




\section{Project W-314 Specific Test and Evaluation Plan 241-AN-A VaTve Pit}

Jack S. Hammers

Numatec Hanford Company, Richland. WA 99352

U.S. Department of Energy Contract DE-AC06-96RL13200

EDT/ECN: 654214

Org Code: $8 \mathrm{C} 610$

UC: 2030

B\&R Code: EW3130010

Charge Code: 106051

Total Pages: 373 9t/5/99

Key Words: Specific Test and Evaluation Plan. Project W-314, 241-AN-A VaTve Pit

TRADEMARK DISCLAIMER. Reference herein to any specific commercial product, process, or service by trade name. trademark, manufacturer, or otherwise, does not necessarily constitute or imply its endorsement. recommendation. or favoring by the United States Government or any agency thereof or its contractors or subcontractors.

Printed in the United States of America. To obtain copies of this document, contact: Document Control Services, P.0. Box 950. Mailstop H6-08. Richland WA 99352. Phone (509) 372-2420: Fax (509) 376-4989.
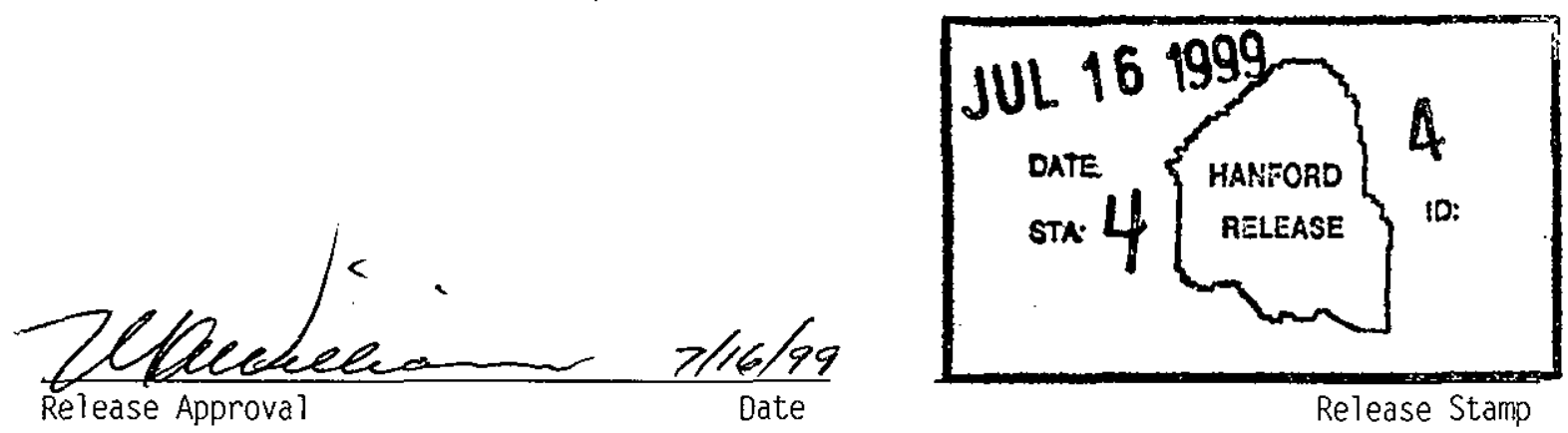

\section{Approved for Public Release}




\section{RECORD OF REVISION}

(1) Document Number

HNF-1733, Rev 4

Page 1

(2) Title

Specific Test and Evaluation Plan for 241-AN-A Valve Pit

CHANGE CONTROL RECORD

(3)

Revision

\begin{tabular}{|c|c|c|c|}
\hline Revision & Pages & $\begin{array}{l}\text { (5) Cog. } \\
\text { Engr. }\end{array}$ & (6) Cog. Mgr. Date \\
\hline 0 & (7) EDT 622787 Dated December 09, 1977 & & \\
\hline 1 & $\begin{array}{l}\text { 241-AN-A Valve Pit Specific Test and } \\
\text { Evaluation Plan. HNF-1733 Delete reference } \\
\text { to Cathodic Protection ATP. Revise Table } \\
5-1 \text { by deleting 225A breaker replacement } \\
\text { work activity and correcting panel numbers } \\
\text { EDS-DP-105 \& EDS-DP-106 to EDS-DP-103 \& } \\
\text { EDS-DP-105 respectively. Add drawing } \\
\text { reference for Construction Acceptance Test } \\
\text { 1.4.C.A.C.15. Renumbered tests to para17e } \\
\text { required tests for each architectural unit. } \\
\text { Updated Logic Diagram. ECN } 643769\end{array}$ & TL Warnick & WH Hays \\
\hline 2 & $\begin{array}{l}\text { ECN } 644838 \text { 241-AN-A Valve Pit Specific Test } \\
\text { \& Evaluation Plan, HNF-1733, Rev. } 2 . \\
\text { Delete reference to Impact Wrench Assembly } \\
\text { Factory Acceptance Test (FAT). Rename } \\
\text { Construction Acceptance Test (CAT) to } \\
\text { Construction Test and Inspection (CTI). } \\
\text { Added test review, approval, and witness } \\
\text { responsibilities. }\end{array}$ & TL Warnick & WH Hays \\
\hline 3 & $\begin{array}{l}\text { ECN } 650509 \text { 241-AN-A Valve Pit Specific Test } \\
\text { and Evaluation Plan. HNF-1733, Rev. } 3 \text {. } \\
\text { Revised Work Scope and Construction Testing } \\
\text { to reflect current project scope. }\end{array}$ & TL Warnick & WH Hays \\
\hline $\begin{array}{l}4 \\
\text { RS }\end{array}$ & $\begin{array}{l}\text { ECN 654214, 241-AN-A Valve Pit Specific } \\
\text { Test and Evaluation Plan, HNF-1733, Rev. } 4 . \\
\text { Revised construction testing to reflect } \\
\text { current project scope. } \\
\text { a } 7 \text {. }\end{array}$ & JL Gruba & $\begin{array}{l}\text { JS Hammers } \\
\text { Hammen }\end{array}$ \\
\hline & & & \\
\hline & & & \\
\hline & & & \\
\hline & & & \\
\hline
\end{tabular}




\title{
PROJECT W-314
}

\section{SPECIFIC TEST AND EVALUATION PLAN}

\author{
241-AN-A VALVE PIT
}


TABLE OF CONTENTS

1.0 PURPOSE

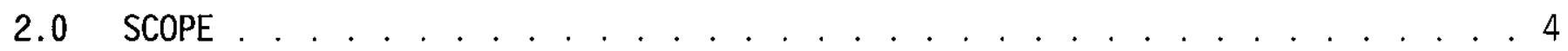

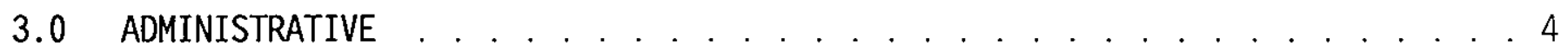

3.1 SAFETY INFORMATION/COMMUNICATIONS . . . . . . . . . . . . . . . . . 4

4.0 GENERAL INFORMATION AND PREREQUISITES . . . . . . . . . . . . . . . . . . 4

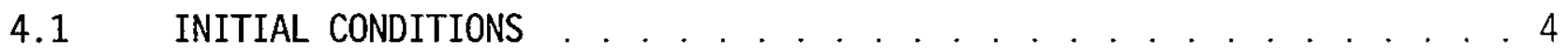

4.2 INSTRUMENTATION AND CONTROL . . . . . . . . . . . . . 5

4.3 FUNCTIONAL TESTS . . . . . . . . . . . . . . . . . 5

4.4 TEMPORARY MODIFICATIONS . . . . . . . . . . . . . . . . 5

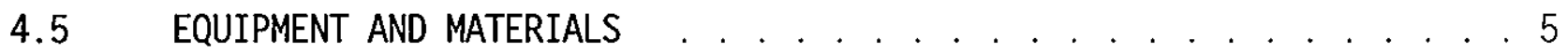

5.0 ASSIGNMENTS OF RESPONSIBILITY . . . . . . . . . . . . . . . . . . . . . . . 5

5.1 JOINT TEST REVIEW GROUP . . . . . . . . . . . . . . 6

5.2 TEST CONFIGURATION CONTROL . . . . . . . . . . . . . 6

6.0 SYSTEM BOUNDARIES . . . . . . . . . . . . . ......... 6

6.1 OBJECTIVE . . . . . . . . . . . . . . . . 6

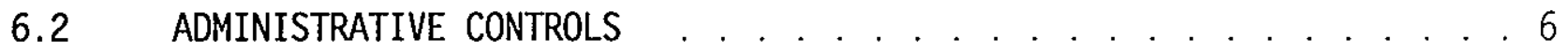

6.3 DESCRIPTION . . . . . . . . . . . . . . . . . . . . . . . . . . . . 7

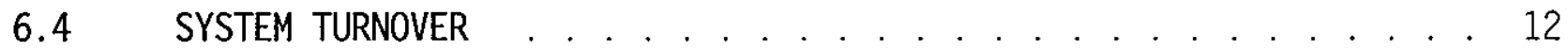

7.0 TEST AND ACCEPTANCE . . . . . . . . . . . . . . . . . . . . . . . . . 12

7.1 VERIFICATION \& VALIDATION . . . . . . . . . . . . . . . . . . . 12

7.2 COMMERCIAL GRADE ITEM DEDICATION . . . . . . . . . . . . . 12

7.3 FACTORY TESTS \& INSPECTIONS . . . . . . . . . . . . . . . . . 13

7.4 CONSTRUCTION TESTS \& INSPECTIONS . . . . . . . . . . . . . . . . . . . 14

7.5 ACCEPTANCE TESTING . . . . . . . . . . . . . . . . . . 18

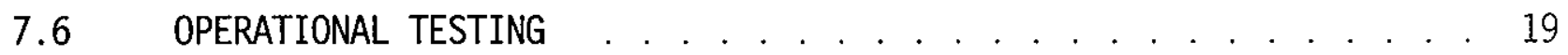

7.7 241-AN-A VALVE PIT TEST COMPLETION . . . . . . . . . . . . 20

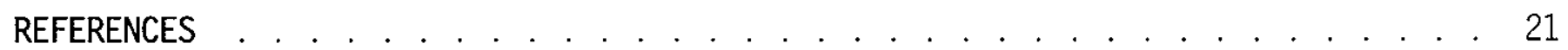

241-AN-A VALVE PIT TEST COMPLETION CHECKLIST . . . . . . . . . . . . . . . . . 23 
TABLE OF CONTENTS (CONT.)

\section{LIST OF TABLES}

TABLE 5-1 241-AN-A VALVE PIT WORK AND TEST BOUNDARIES . . . . . . . . 8

TABLE 7.2 COMMERCIAL GRADE ITEM DEDICATION ACTIVITIES . . . . . . . . . 13

TABLE 7-3 FACTORY TESTS \& INSPECTIONS . . . . . . . . . . . . . . . . . . . 13

TABLE 7.4 CONSTRUCTION TESTS \& INSPECTIONS . . . . . . . . . . . . . . . 14

TABLE $7-5$ ACCEPTANCE TEST PROCEDURES . . . . . . . . . . . . . . . 18

TABLE 7.6 OPERATIONAL TEST PROCEDURES $\ldots \ldots \ldots \ldots$

\section{LIST OF FIGURES}

FIGURE 1

FIGURE 1
241-AN-A VALVE PIT LOGIC DIAGRAM

241-AN-A VALVE PIT LOGIC DIAGRAM 


\subsection{PURPOSE}

The purpose of this Specific Test and Evaluation Plan (STEP) is to provide a detailed written plan for the systematic testing of modifications made to the 241-AN-A Vaive Pit by the $W$-314 Project. The STEP develops the outline for test procedures that verify the system's performance to the established Project design criteria. The STEP is a "lower tier" document based on the W-314 Test \& Evaluation Plan (TEP).

\section{$2.0 \quad$ SCOPE}

This STEP encompasses a 11 testing activities required to demonstrate compliance to the project design criteria as it relates to the modifications of the AN-A valve pit. The Project Design Specifications (PDS) identify the specific testing activities required for the Project. Testing includes Validations and Verifications (e.g., Commercial Grade Item Dedication activities...etc), Factory Tests \& Inspections (FTIs), Construction Tests \& Inspections (CTIS), Acceptance Test Procedures (ATPS), and Operational Test Procedures (OTPS). The STEP will be utilized in conjunction with the TEP for verification and validation.

\subsection{ADMINISTRATIVE}

\subsection{Safety Information/Communications}

Testing and inspection activities will be performed to the safety and communication procedures of the testing facility (e.g., shop, mockup, tank farm, vendor facility....etc.)

During the performance of 241-AN Tank Farm ATPs and OTPS, the Operations Representative Fest Director will be the direct line of communication with the Shift Office. During abnormal and casualty situations, the Double-Shell Tank Farms Shift Manager will be the building emergency director/event commander.

\subsection{GENERAL INFORMATION AND PREREQUISITES}

\subsection{Initial Conditions}

Prerequisite tests and a 11 required construction, specific to the test. shal1 be completed prior to beginning any test. Test boundaries shall be verified as being appropriate and secure. Required locks and tags shall be correctly placed to allow performance of the test without disturbing the lock and tag. Required personnel shall be available and briefed on the steps of the test (a pre-job briefing is 
a prerequisite that must be satisfied before performing a Construction Work Package (CWP). ATP, and/or OTP). Required test apparatus shal1 be functional and set up in a safe configuration for the test. Installed and existing equipment, required for the test, shall be verified to be ready to operate in the test conditions.

\subsection{Measurement \& Test Equipment}

Instruments used will be specified per the test document and controlled per the performing agency's Measurement \& Test Equipment program.

\subsection{Functional Tests}

Functional tests, when required for calibration of equipment, will be performed prior to the associated ATP or OTP. Functional tests will be performed to approved documents.

\subsection{Temporary Modifications}

Temporary modifications may be required in 241-AN Tank Farm. Temporary modifications will be made per HNF-IP-0842, Vol. IV, section 4.5.

\subsection{Equipment and Materials}

Equipment installed by the project that fails during testing, or existing equipment that is damaged by testing, will be the responsibility of the construction contractor. Existing facility SSCS, not damaged but found defective, will be the responsibility of Double-Shel1 Tanks organization. Initiation of repairs will be accomplished by the creation of either a work package, using JCS, or a Construction Work Package (CWP).

\subsection{ASSIGNMENTS OF RESPONSIBILITY}

The design agent (FDNW) will provide test directors for the Acceptance Test Procedures. Tank Farm Operations will provide a qualified Tank Farm Shift Manager or Operations Engineer who will be the Test Directors for all 0TPS. This representative wi11 accept the results of the ATPS for Tank Farm Operations. Roles and responsibilities for construction. TWRS Operations, TFRSO Startup Group. W-314 Project Management, Acceptance Inspection, and Quality Assurance are defined in the W-314 TEP. Chapter three. 


\subsection{Joint Test Review Group}

The JTRG will consist of the Chief Test Director (from the TWRS Testing and Systems Readiness organization), Engineering manager (of the facility that will operate the equipment), W-314 Startup Manager. the Design Authority, Operations, Project QA, applicable Test Director for each ATP \& OTP, and W-314 Project Manager. The JTRG provides Tank Farm Operations with a high level of confidence that the test can be performed safely and efficiently. The review by the JTRG provides a basis for test procedure approval and release. The JTRG may conduct thorough reviews of ATPS and OTPS to ensure compliance with applicable procedural requirements, to ensure they can be performed safely, and to ensure the scope of testing and inspections provide a product that satisfies operational and safety requirements. The JTRG reviews test procedure data, and provides their recommendation concerning the finat acceptance that test results satisfy the design specifications as stated in the ATPS, OTPS, or test plans.

\subsection{Test Configuration Control}

Testing shall be conducted using detailed test procedures and the latest versions of a 11 ECNs and Drawings for the Project.

Configuration control shall conform to HNF-PRO-440 and HNF-PRO-226 HNF-PRO-224. Drawings shal1 be retained in project status by the $W$-314 Project until such time as all work on the 241-AN-A valve pit is complete and accepted.

\subsection{SYSTEM BOUNDARIES}

\subsection{Objective}

Recommended initial system boundaries are identified for use in planning and implementing the various tests listed in this STEP. These system boundaries will provide safe work boundaries which allow testing to proceed in a safe environment. isolated from facility hazards.

\subsection{Administrative Controls}

Where actual physical isolations are not possible (e.g., pit nozzles...etc), Lock and Tag system (HNF-IP-0842, Vol. II, Sections 4.9.1 and 4.10.1), as a minimum, will be utilized to establish the required administrative controls. 
HNF-1733, Rev. 4

\subsection{Description}

The table below lists the work and test boundaries for 241-AN-A valve pit work. These boundaries are estimates and may be modified at the time of execution to match current conditions in the farm and the job. This table will not be modified to reflect those changes. 
HNF-1733, Rev. 4

TABLE 5-1 241-AN-A VALVE PIT WORK AND TEST BOUNDARIES

\begin{tabular}{|c|c|c|c|c|}
\hline WORK ACTIVITY & PROJECT DRAWING & ESSENTIAL ORAWING & LOCK \& TAG LOCATION & COMMENTS \\
\hline 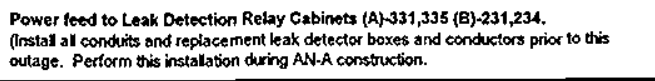 & $\begin{array}{l}\text { H-14-100937 } \\
\mathrm{H}-14-100938\end{array}$ & H-14-030001, sh 11 & Distribution Panel EDS-OP-105, MAIN 8REAKER & \\
\hline 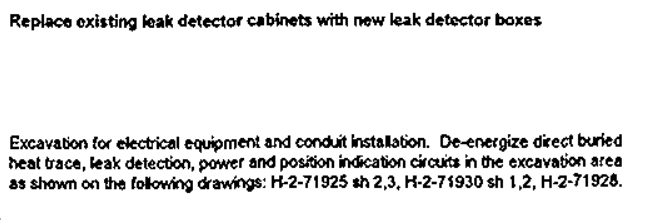 & 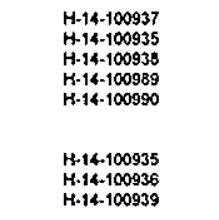 & 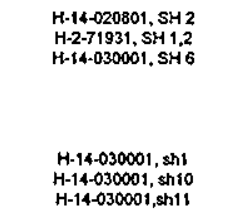 & $\begin{array}{l}\text { OISTRIBUTION PANEL EOS-OP-101, BREAKER it } \\
\text { To be determined based on exact areas to be excavated and resurts of surface scan. }\end{array}$ & 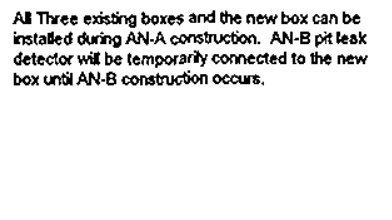 \\
\hline 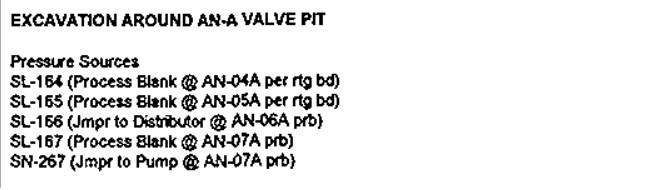 & $\begin{array}{l}H-14-100933 \\
H-14-100941 \\
H-14-100942\end{array}$ & $H-14-020801$, sh 2 & & \\
\hline $\begin{array}{l}\text { Electicol Sources } \\
\text { Heat Trace } \\
\text { SL-164 } \\
\text { SL-165 } \\
\text { SL-166 } \\
\text { SL-167 } \\
\text { SN-267 } \\
\text { Leak Detectors } \\
\text { LDE-VP.AN-A (LOE-113) }\end{array}$ & $\begin{array}{l}\text { N/A } \\
\text { N/R } \\
N / A \\
N / A \\
N / A \\
\text { NAA }\end{array}$ & 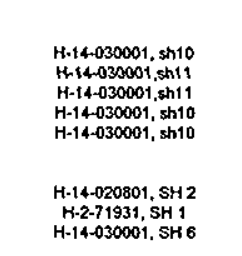 & 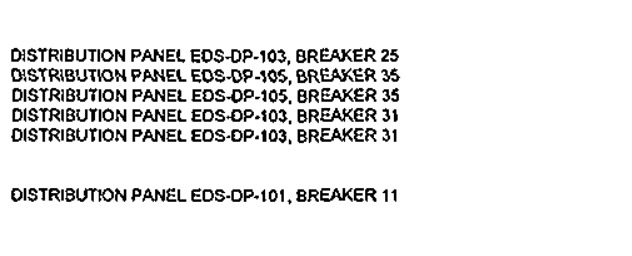 & 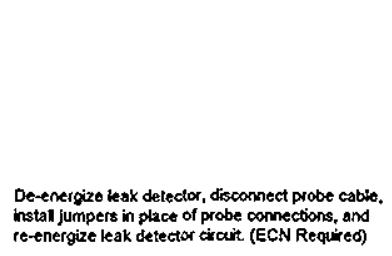 \\
\hline 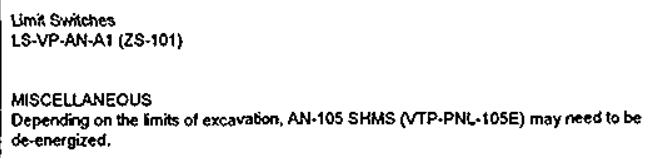 & NAA & $\begin{array}{l}H \cdot 14-030001, \operatorname{sh} 6 \\
H-14-030001, \operatorname{sh} 11\end{array}$ & $\begin{array}{l}\text { DISTRIBUTION PANEL, EDS-DP-101, BREAKER } 6 \\
\text { DISTRIBUTION PANEL EOS-DP-103, BREAKER } 26\end{array}$ & $\begin{array}{l}\text { ECN } 636365 \text { reterences cranges to H-2.71927, sh } \\
\text { 3. }\end{array}$ \\
\hline
\end{tabular}




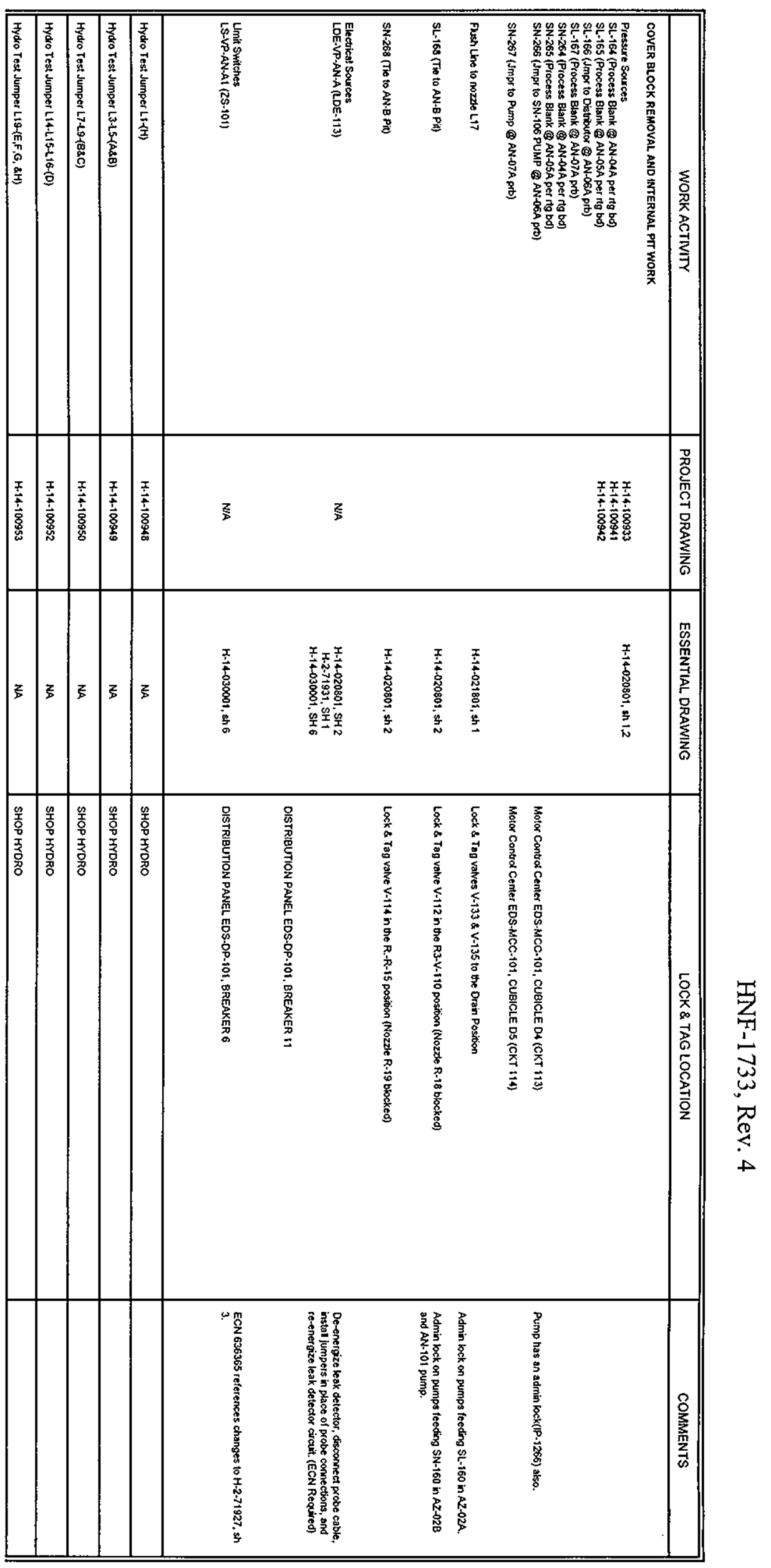




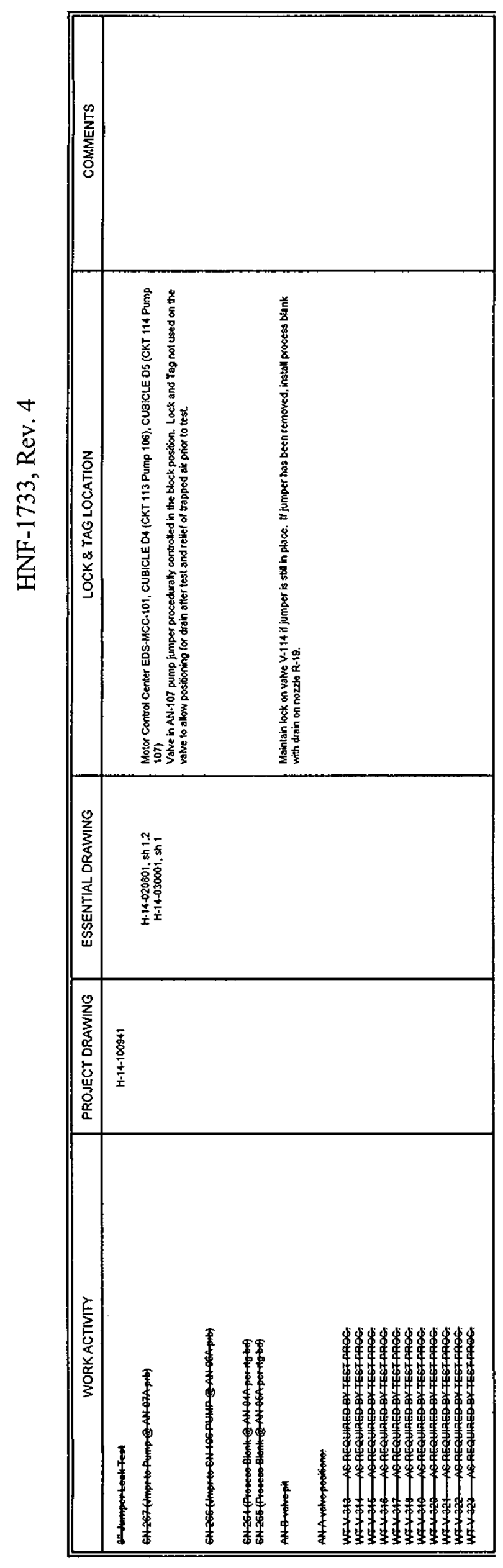

요 


\begin{tabular}{|c|c|c|c|c|}
\hline & 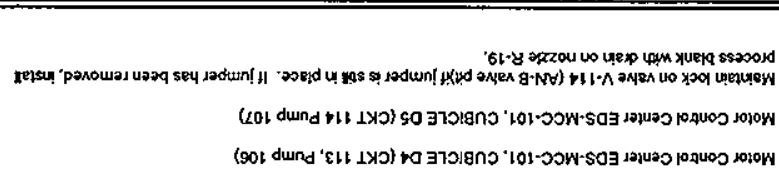 & 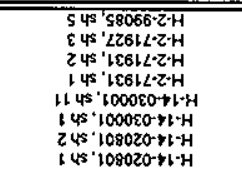 & 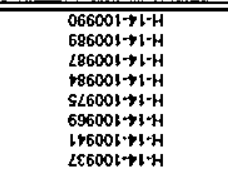 & 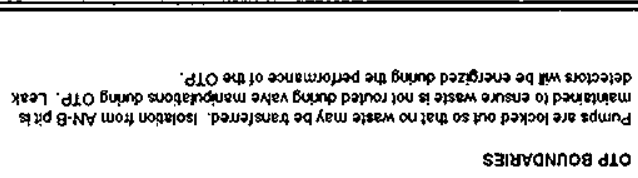 \\
\hline & 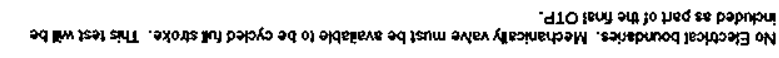 & suon & $\angle 8600 \mathrm{l}-\nabla t \cdot H$ & 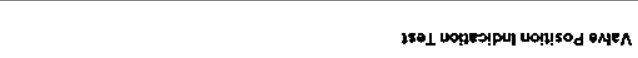 \\
\hline & 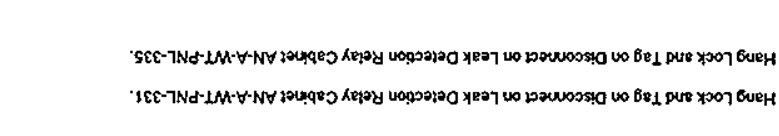 & 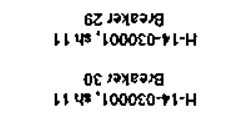 & 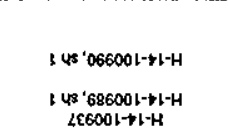 & 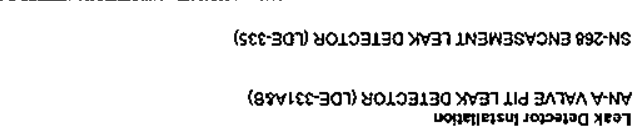 \\
\hline & 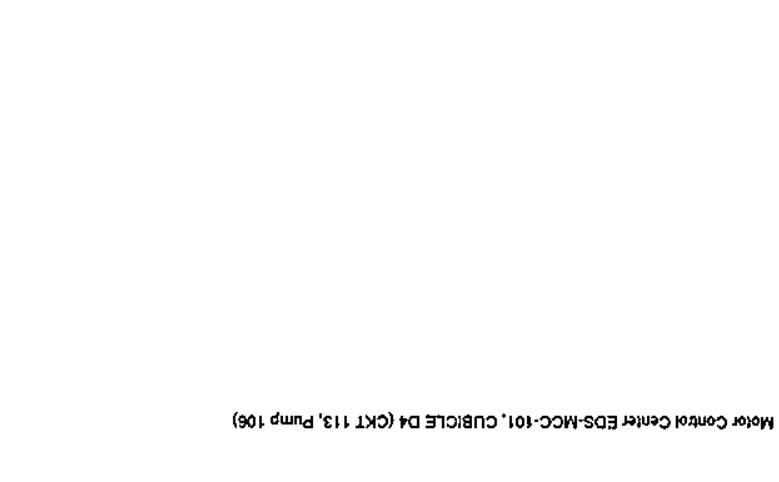 & 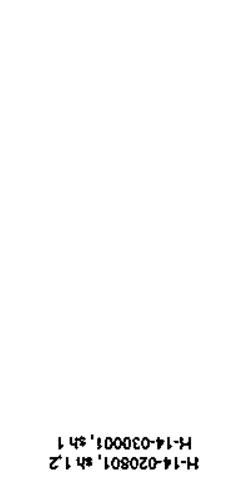 & $|+6000|-\$ 1-H$ & 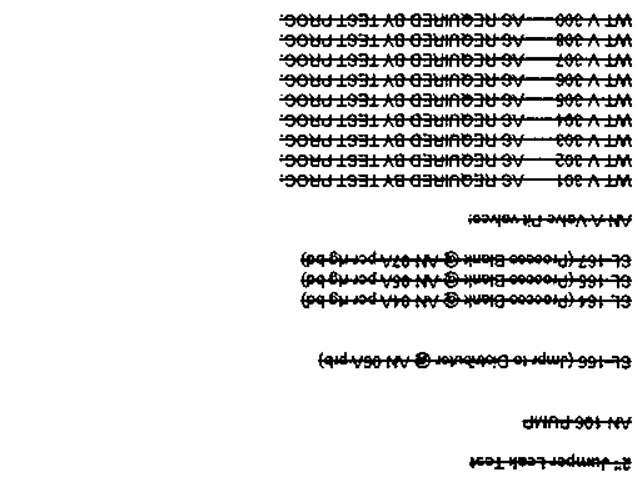 \\
\hline 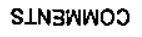 & NOI1 $\forall 0079 \forall 18 \times 501$ & פNIMVAO רVILNSSS & ONIMVZO LOZROYd & ЦLINILОУ ХХХОМ \\
\hline
\end{tabular}


HNF-1733, Rev. 4

\subsection{System Turnover}

Following completion and acceptance of a 11 the required testing and testing data within a given boundary, the systems and equipment in that area will be ready for release to operations for their control and use. The SSC(s) will be released to TWRS Operations by using the Acceptance for Beneficial Use process described in the W-314 TEP (HNF-SD-W314-TEP-001).

\subsection{TEST AND ACCEPTANCE}

The Project $W-314$ 241-AN-A Valve Pit modification STEP includes design verifications performed by various methods including engineering analysis. Vendor data review, FTIs and CTIs performed in accordance with Procurement/Construction Specifications, drawings, and system functional tests performed in accordance with ATPS and OTPS. These verifications and tests wi11 be documented in the $W$-314 Project 241-AN-A Requirements Verification Report (RVR), HNF-1534, Rev.0, and in specific test documentation (e.g., ATRs, OTRs, CWP...etc.). In addition, approved calibration and functional test procedures will be performed in preparation for the above listed tests.

\subsection{Vatidation and Verification}

A11 design requirements listed in the Project Development Specifications are formally reviewed by analysis and examination for project compliance. Commercial grade equipment utilized in Safety Class applications, will be qualified per appropriate procedures and programs. Equipment used in non-safety class applications will be verified using vendor data to ensure the design requirements of the application are met. These reviews are documented in the 241-AN-A Valve Pit RVR. This report is listed in the reference section of this document.

\subsection{Commercial Grade Item Dedication}

The following table lists the testing that will be performed to satisfy the Commercial Grade Item Dedication requirements for the modification of 241-AN-A Valve Pit. Note that acceptance criteria are included in the individual drawings, specifications, procedures, and CWPS. 
TABLE 7-2

\begin{tabular}{|c|c|c|c|c|c|}
\hline TEST NUMBER & $\begin{array}{l}\text { TEST } \\
\text { NAME }\end{array}$ & $\begin{array}{l}\text { REFERENCE } \\
\text { DOCUMENT }\end{array}$ & LOCATION & $\begin{array}{l}\text { TEST } \\
\text { DOCUMENT }\end{array}$ & INTERDEPENDENCIES \\
\hline 1.4.C.A.CG.1 & $\begin{array}{l}\text { Rebar Tensile Test } \\
\text { and Physical } \\
\text { Inspection }\end{array}$ & $\begin{array}{l}\text { W-314-C1, section } \\
03300,1.5 .2 .1\end{array}$ & Shop & & $\begin{array}{l}\text { Test reports must be in place prior } \\
\text { to installing any Rebar. }\end{array}$ \\
\hline 1.4.C.A.CG. 2 & $\begin{array}{l}\text { Steel Plate, bar and } \\
\text { shape Tensile test } \\
\text { and physical } \\
\text { Inspection }\end{array}$ & $\begin{array}{l}\text { W-314-C1, section } \\
05500,1.3 .2 .1\end{array}$ & Shop & & $\begin{array}{l}\text { Test reports must be in place prior } \\
\text { to installing any items for which } \\
\text { testing was required. }\end{array}$ \\
\hline 1.4.C.A.CG.3 & $\begin{array}{l}\text { Commercial Grade } \\
\text { Dedication for } \\
\text { instrumentation }\end{array}$ & $\begin{array}{l}\text { W-314-C1, section } \\
13440,2.3 .2\end{array}$ & Shop & & $\begin{array}{l}\text { Tests must be documented as } \\
\text { acceptable prior to installing any } \\
\text { components in field locations }\end{array}$ \\
\hline 1.4.C.A.CG & $\begin{array}{l}\text { Commercial Grade } \\
\text { Dedication for } \\
\text { Piping Materials, } \\
\text { verify pipe size }\end{array}$ & $\begin{array}{l}\text { W-314-C1, section } \\
15493,1.2 .2 .1 \text { and } \\
2.4 ; \text { PDS-001 } \\
3.2 .2 .6\end{array}$ & Shop & & $\begin{array}{l}\text { Test Reports must be accepted } \\
\text { prior to fabrication of piping spools. }\end{array}$ \\
\hline 1.4.C.A.CG.5 & $\begin{array}{l}\text { Commercial Grade } \\
\text { Dedication for } \\
\text { Electrical. }\end{array}$ & $\begin{array}{l}\text { W-314-C3, section } \\
16400,2.4 .1\end{array}$ & Shop & & $\begin{array}{l}\text { Test Reports must be accepted } \\
\text { prior to installing any components } \\
\text { or parts in fabricated assemblies or } \\
\text { field locations. }\end{array}$ \\
\hline
\end{tabular}

\subsection{Factory Tests \& Inspections}

The following table lists the FTIs that will be performed to modify 241-AN-A Valve Pit. Note that acceptance criteria are included in the individual drawings, specifications, procedures, and CWPS.

TABLE $7 \cdot 3$

\begin{tabular}{|c|l|l|l|l|l|l}
\hline \begin{tabular}{|l}
\hline TEST \\
NUMBER
\end{tabular} & $\begin{array}{l}\text { TEST } \\
\text { NAME }\end{array}$ & $\begin{array}{l}\text { REFERENCE } \\
\text { DOCUMENT }\end{array}$ & LOCATION & $\begin{array}{l}\text { TEST } \\
\text { DOCUMENT }\end{array}$ & INTERDEPENDENCIES & $\begin{array}{l}\text { 1) REVIEW, 2) APPROVAL, \& } \\
\text { 3) WITNESS } \\
\text { RESPONSIBILITIES }\end{array}$ \\
\hline 1.4.C.A.F.1 & $\begin{array}{l}\text { Valve Hydro and } \\
\text { Leak tests }\end{array}$ & W-314-P1, section 3.9 & Factory & & Prior to shipping & $\begin{array}{l}\text { Design Agent - 1,2,3 } \\
\text { Design Authority - 1, 2,3 }\end{array}$ \\
\hline
\end{tabular}




\subsection{Construction Tests \& Inspections}

The table below lists the construction tests performed during fabrication, modification and installation to verify functionality of structures, systems and components following installation. AI inspection activities performed during construction are referenced in "ACCEPTANCE INSPECTION PLAN", W-314-C1-1, REV. B. Note that acceptance criteria are included in the individual test drawings. specifications, procedures, and CWPS.

TABLE 7-4

\begin{tabular}{|c|c|c|c|c|c|c|}
\hline \multicolumn{7}{|c|}{ 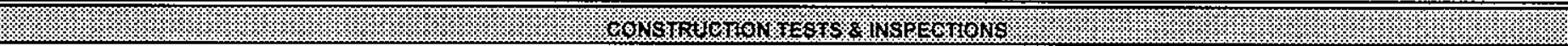 } \\
\hline TEST & TEST Th & REFERENCE DOCUMENT & LOCATON & $\begin{array}{l}\text { OTEST } \\
\text { DOCUMENT }\end{array}$ & INTERDEPENDENCIES & $\begin{array}{l}\text { 1) REVIEW } \\
\text { 2) APPROVAL \& } \\
\text { 3) WITNESS } \\
\text { RESPONSIBILITIES. }\end{array}$ \\
\hline 1.4.C.A.C.1 & In-Place Density Test & $\begin{array}{l}\text { W-314-C1, section } 02220 \text {, } \\
\text { 3.1.6.2 }\end{array}$ & Field & CWP & $\begin{array}{l}\text { Required prior to } \\
\text { excavation for all } \\
\text { excavations }\end{array}$ & QC-3 \\
\hline 1.4.C.A.C. 2 & $\begin{array}{l}\text { Backfill Density Test \& } \\
\text { Cont. Soil Use }\end{array}$ & $\begin{array}{l}\text { W-314-C1, section 02220, 3.3, } \\
\text { 3.4, 3.6.1; PDS-001, 3.3.1.6, } \\
\text { 3.3.1.7 }\end{array}$ & Field & CWP & During \& after backfill & QC-3 \\
\hline 1.4.C.A.C.3 & Concrete Slump Test & $\begin{array}{l}\text { W-314-C1, section } 03300 \text {, } \\
\text { 4.5.2.4, 3.4.1; PDS-001, } \\
\text { 3.3.1.4; PDS-002.3.3.4.2 }\end{array}$ & Field & CWP & $\begin{array}{l}\text { During concrete } \\
\text { placement }\end{array}$ & QC-3 \\
\hline 1.4.C.A.C.4 & Concrete Air Content Test & $\begin{array}{l}\text { W-314-C1, section 03300, } \\
4.5 .2 .4 ; \text { PDS-001 3.3.1.4; } \\
\text { PDS-002, 3.3.4.2 }\end{array}$ & Field & CWP & $\begin{array}{l}\text { During concrete } \\
\text { placement }\end{array}$ & QC-3 \\
\hline 1.4.C.A.C.5 & Concrete Test Cylinders & $\begin{array}{l}\text { W-314-C1, section 03300, } \\
4.5 .2 .4 ; \text { PDS-001 3.3.1.4; } \\
\text { PDS-002,3.3.4.2 }\end{array}$ & Field, Lab & $\begin{array}{l}\text { Testing Agency } \\
\text { Report }\end{array}$ & $\begin{array}{l}\text { Cast cylinders during } \\
\text { concrete placement }\end{array}$ & QC-3 \\
\hline 1.4.C.A.C.6 & $\begin{array}{l}\text { Concrete Replacement } \\
\text { Inspections }\end{array}$ & $\begin{array}{l}\text { W-314-C1, section } 03300,3.1, \\
3.2 .1,3.2 .2 .1, \& 3.4 .2 ; \\
\text { PDS-001, 3.3.1.4; PDS-002 } \\
3.3 .4 .2,-3.7 .3 .2 .4\end{array}$ & Field & CWP & Prior to Placing Concrete & QC-3 \\
\hline 1.4.C.A.C.7 & $\begin{array}{l}\text { Concrete Placement } \\
\text { Inspections }\end{array}$ & $\begin{array}{l}\text { W-314-C1, section } 03300,3.2 \\
\& 3.4 .2 ; \text { PDS-001, 3.3.1.4; } \\
\text { PDS-002, 3.3.4.2 }\end{array}$ & Field & CWP & $\begin{array}{l}\text { During concrete } \\
\text { placement }\end{array}$ & QC-3 \\
\hline 1.4.C.A.C.8 & $\begin{array}{l}\text { Concrete Post-Placement } \\
\text { Inspections }\end{array}$ & $\begin{array}{l}\text { W-314-C1, section } 03300,3.2 \text {, } \\
3.3 \& 3.4 .2\end{array}$ & Field & CWP & $\begin{array}{l}\text { Prior to Cover block } \\
\text { Installation. }\end{array}$ & QC-3 \\
\hline 1.4.C.A.C.9 & $\begin{array}{l}\text { Expansion Anchor } \\
\text { Inspections }\end{array}$ & $\begin{array}{l}\text { W-314-C1, section } 05055 \text {, } \\
\text { 3.4.1 }\end{array}$ & Field & CWP & $\begin{array}{l}\text { During anchor } \\
\text { installation }\end{array}$ & QC-3 \\
\hline 1.4.C.A.C. 10 & $\begin{array}{l}\text { Structural Weld } \\
\text { Examinations }\end{array}$ & $\begin{array}{l}\text { W-314-C1, section } 05500 \text {, } \\
3.4 .1\end{array}$ & Shop, Field & CWP & $\begin{array}{l}\text { Prior to coating any } \\
\text { structural pieces. }\end{array}$ & QC-3 \\
\hline 1.4.C.A.C.11 & $\begin{array}{l}\text { Cover Block Special } \\
\text { Protective Coating Surface } \\
\text { Preparation Inspection }\end{array}$ & $\begin{array}{l}\text { W-314-C1, section } 09855 \\
3.2 .2 \text { \& 3.4.2; PDS-005, } \\
3.3 .1 .2 .1,3.2 .2 .6\end{array}$ & Shop, Field & CWP & $\begin{array}{l}\text { Prior to application of } \\
\text { coatings }\end{array}$ & QC-3 \\
\hline 1.4.C.A.C.12 & $\begin{array}{l}\text { Cover Block Special } \\
\text { Protective Coating } \\
\text { Application }\end{array}$ & $\begin{array}{l}\text { W-314-C1, section 09855, } \\
3.4 .1 ; \text { PDS-005, 3.2.2.1, } \\
3.2 .2 .2,3.2 .2 .8,3.2 .4,3.3 .1 .2 .2\end{array}$ & Shop, Field & CWP & $\begin{array}{l}\text { During application of } \\
\text { each coat }\end{array}$ & QC-3 \\
\hline 1.4.C.A.C.13 & $\begin{array}{l}\text { Pit Special Protective } \\
\text { Coating Surface } \\
\text { Preparation Inspection }\end{array}$ & $\begin{array}{l}\text { W-314-C1, section 09855, } \\
\text { 3.2.2 \& 3.4.2; PDS-005, } \\
\text { 3.3.1.2.1, 3.2.2.6 }\end{array}$ & Shop, Field & $C W P$ & $\begin{array}{l}\text { Prior to application of } \\
\text { coatings }\end{array}$ & QC-3 \\
\hline
\end{tabular}


HNF-1733, Rev. 4

\begin{tabular}{|c|c|c|c|c|c|c|}
\hline \multicolumn{7}{|c|}{ 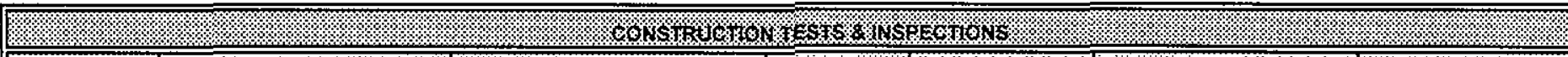 } \\
\hline $\begin{array}{l}\text { TEST } \\
\text { NUMBER } \\
\end{array}$ & 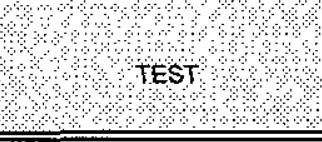 & REFERENCE DOCUMENT & locAtion & $\begin{array}{l}\text { की } \\
\text { DOSTS }\end{array}$ & w N ERDEPENDENSIES & $\begin{array}{l}\text { 1) REVEW } \\
\text { 2) APPROVAL \& } \\
\text { 3) WITNESS } \\
\text { RESPONSIBILITIES }\end{array}$ \\
\hline 1.4.C.A.C.14 & $\begin{array}{l}\text { Pit Special Protective } \\
\text { Coating Application }\end{array}$ & $\begin{array}{l}\text { W-314-C1, section 09855, } \\
3.4 .1 ; \text { PDS-005 3.2.2.1, } \\
3.2 .2 .2,3.2 .2 .8,3.2 .4,3.3 .1 .2 .2\end{array}$ & Shop, Field & CWP & $\begin{array}{l}\text { During application of } \\
\text { each coat }\end{array}$ & QC-3 \\
\hline 1.4.C.A.C.15 & $\begin{array}{l}\text { Cover Block Painting } \\
\text { Diagrams }\end{array}$ & $\begin{array}{l}\text { W-314-C1, section } 09855 \text {, } \\
\text { 3.3.6 \& 3.4.2; PDS-002, } \\
3.7 .3 .2 .53 .3 .3 .1\end{array}$ & Shop & CWP & $\begin{array}{l}\text { Verify painting diagram } \\
\text { matches information } \\
\text { given in drawing } s \\
\text { referenced in section } \\
\text { 3.3.6. }\end{array}$ & QC-3 \\
\hline 1.4.C.A.C.16 & $\begin{array}{l}\text { Leak Detector Fabrication } \\
\text { Wiring Continuity }\end{array}$ & $\begin{array}{l}\text { W-314-C1, section 13440, } \\
\text { 3.2.1.1 \& section } 16400 \text {. } \\
\text { 3.3.1.1 }\end{array}$ & Shop, Field & CWP & $\begin{array}{l}\text { Complete prior to } \\
\text { performing ATP. }\end{array}$ & QC-3 \\
\hline 1.4.C.A.C.17 & $\begin{array}{l}\text { Leak Detector Cabinet } \\
\text { Installation Wiring } \\
\text { Continuity }\end{array}$ & $\begin{array}{l}\text { W-314-C1, section } 13440, \\
3.2 .1 .1 \text { \& section } 16400, \\
3.3 .1 .1\end{array}$ & Shop, Field & CWP & $\begin{array}{l}\text { Complete prior to } \\
\text { performing ATP. }\end{array}$ & QC-3 \\
\hline 1.4.C.A.C.18 & $\begin{array}{l}\text { Cover Block installation } \\
\text { Wiring Continuity }\end{array}$ & $\begin{array}{l}\text { W-314-C1, section } 13440 \text {, } \\
\text { 3.2.1.1 \& section } 16400 \text {, } \\
\text { 3.3.1.1 }\end{array}$ & Shop, Field & CWP & $\begin{array}{l}\text { Complete prior to } \\
\text { performing ATP. }\end{array}$ & QC-3 \\
\hline 1.4.C.A.C.19 & $\begin{array}{l}\text { Terminal Box Conduit \& } \\
\text { Cable Installation Wiring } \\
\text { Continuity }\end{array}$ & $\begin{array}{l}\text { W-314-C1, section } 13440 \text {, } \\
\text { 3.2.1.1 \& section } 16400 \text {, } \\
\text { 3.3.1.1 }\end{array}$ & Shop, Field & CWP & $\begin{array}{l}\text { Complete prior to } \\
\text { performing ATP. }\end{array}$ & QC-3 \\
\hline 1.4.C.A.C.20 & $\begin{array}{l}\text { Valve Limit Switch Wiring } \\
\text { Continuity }\end{array}$ & $\begin{array}{l}\text { W-314-C1, section } 13440 \text {, } \\
\text { 3.2.1.1 \& section } 16400 \text {, } \\
\text { 3.3.1.1 }\end{array}$ & Shop, Field & CWP & $\begin{array}{l}\text { Complete prior to } \\
\text { performing ATP. }\end{array}$ & QC-3 \\
\hline 1.4.C.A.C.21 & $\begin{array}{l}\text { Leak Detector Installation } \\
\text { Wiring Continuity }\end{array}$ & $\begin{array}{l}\text { W-314-C1, section 13440, } \\
3.2 .1 .1 \text { \& section } 16400 \\
3.3 .1 .1\end{array}$ & Shop, Field & CWP & $\begin{array}{l}\text { Complete prior to } \\
\text { performing ATP. }\end{array}$ & QC-3 \\
\hline 1.4.C.A.C. 22 & $\begin{array}{l}\text { Cover Block Wiring } \\
\text { Shielding Resistance to } \\
\text { Ground }\end{array}$ & $\begin{array}{l}\text { W-314-C1, section } 13440 \text {, } \\
\text { 3.2.1.2, } \mathrm{H}-14-100936 \mathrm{SH} 3 \\
\text { NOTE } 24\end{array}$ & Field & CWP & $\begin{array}{l}\text { Complete prior to } \\
\text { performing ATP }\end{array}$ & QC-3 \\
\hline 1.4.C.A.C.23 & $\begin{array}{l}\text { Terminal Box Conduit \& } \\
\text { Cable Shielding Resistance } \\
\text { to Ground }\end{array}$ & $\begin{array}{l}\text { W-314-C1, section 13440, } \\
\text { 3.2.1.2 }\end{array}$ & Field & CWP & $\begin{array}{l}\text { Complete prior to } \\
\text { performing ATP }\end{array}$ & QC-3 \\
\hline 1.4.C.A.C. 24 & $\begin{array}{l}\text { Valve Limit Switch } \\
\text { Shielding Resistance to } \\
\text { Ground }\end{array}$ & $\begin{array}{l}\text { W-314-C1, section } 13440 \text {, } \\
3.2 .1 .2\end{array}$ & Field & CWP & $\begin{array}{l}\text { Complete prior to } \\
\text { performing ATP }\end{array}$ & QC-3 \\
\hline 1.4.C.A.C. 25 & $\begin{array}{l}\text { Leak Detector Shielding } \\
\text { Resistance to Ground }\end{array}$ & $\begin{array}{l}\text { W-314-C1, section 13440, } \\
\text { 3.2.1.2 }\end{array}$ & Field & CWP & $\begin{array}{l}\text { Complete prior to } \\
\text { performing ATP }\end{array}$ & QC-3 \\
\hline 1.4.C.A.C. 26 & $\begin{array}{l}\text { Leak Detector Fabrication } \\
\text { Voltage Verification }\end{array}$ & $\begin{array}{l}\text { W-314-C1, section } 16400 \text {, } \\
\text { 3.3.1.2 }\end{array}$ & Field & CWP & $\begin{array}{l}\text { Prior to energizing } \\
\text { equipment. }\end{array}$ & QC-3 \\
\hline 1.4.C.A.C. 27 & $\begin{array}{l}\text { Leak Detector Installation } \\
\text { Voltage Verification }\end{array}$ & $\begin{array}{l}\text { W-314-C1, section } 16400 \text {, } \\
\text { 3.3.1.2 }\end{array}$ & Field & CWP & $\begin{array}{l}\text { Prior to energizing } \\
\text { equipment. }\end{array}$ & QC-3 \\
\hline 1.4.C.A.C. 28 & $\begin{array}{l}\text { Holiday Testing of Piping } \\
\text { Protective Coatings }\end{array}$ & $\begin{array}{l}\text { W-314-C1, section 15493, } \\
\text { 3.1.6.3 }\end{array}$ & Field & CWP & Prior to Backfill. & QC-3 \\
\hline 1.4.C.A.C. 29 & $\begin{array}{l}\text { Piping Fabrication Welding } \\
\text { Non-Destructive } \\
\text { Examination }\end{array}$ & $\begin{array}{l}\text { W-314-C1, section 15493, } \\
\text { 3.2.1 \& Appendix A, 3.3 \& 4.1; } \\
\text { PDS-001, 3.2.2.4, 3.3.1.5, } \\
\text { 3.3.4; PDS-002, 3.3.4.1 }\end{array}$ & Shop, Field & CWP & $\begin{array}{l}\text { Prior to any form of } \\
\text { pressure testing. }\end{array}$ & QC-3 \\
\hline 1.4.C.A.C. 30 & $\begin{array}{l}\text { Jumper Fabrication } \\
\text { Welding Non-Destructive } \\
\text { Examination }\end{array}$ & $\begin{array}{l}\text { W-314-C1, section 15493, } \\
\text { 3.2.1 \& Appendix A, 3.3 \& 4.1; } \\
\text { PDS-001, 3.3.1.5, 3.3.4; } \\
\text { PDS-002, 3.3.4.1 }\end{array}$ & Shop, Field & CWP & $\begin{array}{l}\text { Prior to any form of } \\
\text { pressure testing. }\end{array}$ & QC-3 \\
\hline 1.4.C.A.C.31 & $\begin{array}{l}\text { Piping Fabrication } \\
\text { Cleanliness Inspection }\end{array}$ & $\begin{array}{l}\text { W-314-C1, section } 15493 \text {, } \\
\text { 3.2.3.5; PDS-001, } 3.3 .4\end{array}$ & Shop Field & CWP & $\begin{array}{l}\text { Prior to any form of } \\
\text { pressure testing }\end{array}$ & $Q C-3$ \\
\hline
\end{tabular}


HNF-1733, Rev. 4

\begin{tabular}{|c|c|c|c|c|c|c|}
\hline & & 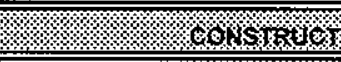 & 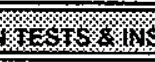 & 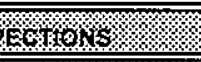 & & \\
\hline $\begin{array}{l}\text { कि } \\
\text { TEST } \\
\mathrm{NUMBER} \\
\end{array}$ & 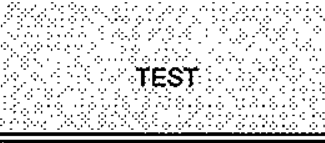 & 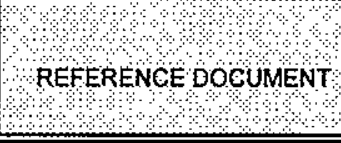 & locAtron & ooCUMENT & का & $\begin{array}{l}\text { 1) REVEW, } \\
\text { 2) APPROVAL, } \\
\text { 3) WITNESS } \\
\text { RESPONSIBILITIES }\end{array}$ \\
\hline 1.4.C.A.C.32 & $\begin{array}{l}\text { Piping Fabrication Pressure } \\
\text { Tests }\end{array}$ & $\begin{array}{l}\text { W-314-C1, section } 15493 \text {, } \\
\text { 3.2.4; PDS-001, 3.3.1.2 }\end{array}$ & Shop Field & CWP & $\begin{array}{l}\text { Test Nozzle spools prior } \\
\text { to installation in the field. }\end{array}$ & $\begin{array}{l}\text { QC-3 } \\
\text { Lead Test Engr -3 }\end{array}$ \\
\hline 1.4.C.A.C.33 & $\begin{array}{l}\text { Piping installation } \\
\text { Cleanliness Inspection }\end{array}$ & $\begin{array}{l}\text { W-314-C1, section 15493, } \\
\text { 3.2.3.5; PDS-001, 3.3.4 }\end{array}$ & Shop Field & CWP & $\begin{array}{l}\text { Prior to any form of } \\
\text { pressure testing }\end{array}$ & QC-3 \\
\hline 1.4.C.A.C.34 & $\begin{array}{l}\text { Piping Installation Pressure } \\
\text { Tests }\end{array}$ & $\begin{array}{l}\text { W-314-C1, section } 15493 \text {, } \\
\text { 3.2.4; PDS-001, 3.3.1.2 }\end{array}$ & Shop Field & CWP & $\begin{array}{l}\text { Test Nozzle spools prior } \\
\text { to installation in the field. }\end{array}$ & $\begin{array}{l}\text { QC-3 } \\
\text { Lead Test Engr -3 }\end{array}$ \\
\hline 1.4.C.A.C. 35 & Jumper Pressure Tests & $\begin{array}{l}\text { W-314-C1, section } 15493 \text {, } \\
\text { Appendix A, 3.6 \& } 4.3\end{array}$ & Shop & CWP & $\begin{array}{l}\text { Test all Jumpers prior to } \\
\text { installation in the valve } \\
\text { pit }\end{array}$ & $\begin{array}{l}\text { QC-3 } \\
\text { Lead Test Engr -3 }\end{array}$ \\
\hline 1.4.C.A.C.36 & $\begin{array}{l}\text { Jumper Fabrication } \\
\text { Inspections }\end{array}$ & $\begin{array}{l}\text { W-314-C1, section 15493, } \\
\text { Appendix A, } 4.2\end{array}$ & Shop & CWP & Prior to shop hydro & QC-3 \\
\hline 1.4.C.A.C.37 & $\begin{array}{l}\text { Jumper in service Leak } \\
\text { Test \& Pit Configuration } \\
\text { Verification }\end{array}$ & $\begin{array}{l}\text { HNF-SD-BIO-001, section } \\
\text { 5.3.2.18, TSR-006, AC } \\
\text { 5.12.2.a }\end{array}$ & Field & $\begin{array}{l}\text { CWP and Leak } \\
\text { Test Procedure }\end{array}$ & $\begin{array}{l}\text { Installation of all jumpers } \\
\text { and required changes at } \\
\text { associated tank central } \\
\text { pump pits. }\end{array}$ & $\begin{array}{l}\text { QC-3 } \\
\text { Lead Test Engr }-2,3 \\
\text { Operations }-2,3 \\
\text { Cog. Engr. }-2\end{array}$ \\
\hline 1.4.C.A.C.38 & $\begin{array}{l}\text { Leak Detector Cabinet } \\
\text { Installation Electrical \& } \\
\text { Instrumentation } \\
\text { Configuration \& Code } \\
\text { Compliance }\end{array}$ & $\begin{array}{l}\text { PDS-001, 3.3.1.1, 3.3.1.10, } \\
3.3 .1 .11,3.3 .1 .12, \text { PDS-003 } \\
3.3 .1 .1,3.3 .1 .2,3.3 .1 .3\end{array}$ & Field & CWP & Prior to performing OTP & NEC Insp. -3 \\
\hline 1.4.C.A.C.39 & $\begin{array}{l}\text { Terminal Box Conduit \& } \\
\text { Cable Installation Electrical } \\
\text { \& Instrumentation } \\
\text { Configuration \& Code } \\
\text { Compliance }\end{array}$ & $\begin{array}{l}\text { POS-001, 3.3.1.1 3.3.1.10, } \\
3.3 .1 .11,3.3 .1 .12, \text { PDS-003 } \\
3.3 .1 .1,3.3 .1 .2,3.3 .1 .3\end{array}$ & Field & CWP & Prior to performing OTP & NEC insp. -3 \\
\hline 1.4.C.A.C. 40 & $\begin{array}{l}\text { Leak Detector Installation } \\
\text { Electrical \& Instrumentation } \\
\text { Configuration \& Code } \\
\text { Compliance }\end{array}$ & $\begin{array}{l}\text { PDS-001, 3.3.1.1, 3.3.1.10, } \\
3.3 .1 .11,3.3 .1 .12, \text { PDS-003 } \\
3.3 .1 .1,3.3 .1 .2,3.3 .1 .3\end{array}$ & Field & CWP & Prior to performing OTP & NEC Insp.-3 \\
\hline 1.4.C.A.C.41 & $\begin{array}{l}\text { Valve Limit Switch } \\
\text { Installation Electrical \& } \\
\text { Instrumentation } \\
\text { Configuration \& Code } \\
\text { Compliance }\end{array}$ & $\begin{array}{l}\text { PDS-001, 3.3.1.1, 3.3.1.10, } \\
3.3 .1 .11,3.3 .1 .12, \text { PDS-003 } \\
3.3 .1 .2\end{array}$ & Field & CWP & Prior to performing OTP & NEC Insp.-3 \\
\hline 1.4.C.A.C. 42 & $\begin{array}{l}\text { Piping Installation } \\
\text { Configuration Verification }\end{array}$ & $\begin{array}{l}\text { PDS-001, 3.2.2.1, 3.2.2.6, } \\
3.2 .2 .7,3.2 .2 .8,3.2 .2 .9 \\
3.2 .2 .10,3.3 .1 .8\end{array}$ & Shop, Field & CWP & $\begin{array}{l}\text { Prior to installation of the } \\
\text { wall nozzles }\end{array}$ & QC-3 \\
\hline 1.4.C.A.C. 43 & Cathodic Protection & $\begin{array}{l}\text { W-314-C1, section 16640, } \\
\text { 3.3.1, 3.3.2 }\end{array}$ & Field & CWP & $\begin{array}{l}\text { After nozzle installation } \\
\text { and prior to application } \\
\text { of protective coating on } \\
\text { pipe }\end{array}$ & QC-3 \\
\hline 1.4.C.A.C. 44 & Jumper Fit Up \& Installation & $\begin{array}{l}\text { TWRS Operations } \\
\text { Requirement }\end{array}$ & Field & $\begin{array}{l}\text { CWP } \\
\text { JCS }\end{array}$ & $\begin{array}{l}\text { TWRS Operations } \\
\text { witness balance \& } \\
\text { installation }\end{array}$ & Operations $-2,3$ \\
\hline 1.4.C.A.C. 45 & $\begin{array}{l}\text { Cover Block Fit \& } \\
\text { Installation }\end{array}$ & $\begin{array}{l}\text { TWRS Operations } \\
\text { Requirement; POS- } 002 \text {, } \\
3.3 .3 .2 .5\end{array}$ & Field & $\begin{array}{l}\text { CWP } \\
\text { JCS }\end{array}$ & $\begin{array}{l}\text { TWRS Operations } \\
\text { witness cover block fit, } \\
\text { balance, \& installation }\end{array}$ & $\begin{array}{l}\text { QC-3 } \\
\text { Operations }-2,3\end{array}$ \\
\hline 1.4.C.A.C. 46 & $\begin{array}{l}\text { Load test Cover Block lifting } \\
\text { bails \& Lifting bail Magnetic } \\
\text { Particle Test }\end{array}$ & $\begin{array}{l}\text { W-314-C1, 03300, section } \\
3.2 .5 ; \text { PDS-002, 3.3.4.2, } \\
3.7 .3 .2 .4\end{array}$ & Shop & CWP & $\begin{array}{l}\text { Prior to Special } \\
\text { Protective Coating } \\
\text { Application/lnstallation. } \\
\text { Lifting bail Mag. Particle } \\
\text { test must be performeo } \\
\text { AFTER load test. }\end{array}$ & QC-3 \\
\hline
\end{tabular}




\begin{tabular}{|c|c|c|c|c|c|c|}
\hline \multicolumn{7}{|c|}{ 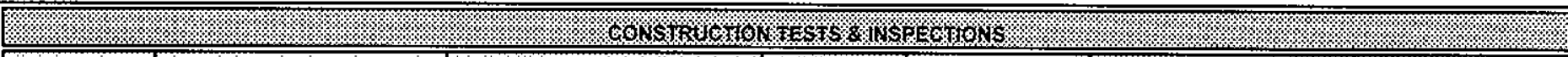 } \\
\hline TTEST & 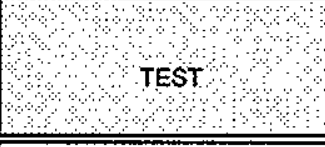 & की & locATon & $\begin{array}{l}\text { के } \\
\text { TEST } \\
\text { boCUMENT }\end{array}$ & נNAERDEPNDENCIES & $\begin{array}{l}\text { 1) REVEW } \\
\text { 2) APPROVAL \& } \\
\text { 3) WITNESS } \\
\text { RESRONSIBILITIES }\end{array}$ \\
\hline 1.4.C.A.C. 47 & $\begin{array}{l}\text { Jumper support leg } \\
\text { adjustment }\end{array}$ & $\begin{array}{l}\text { H-14-100948, Note 7 } \\
\text { H-14-100949, SH1, Note 7 } \\
\text { H-14-100950, SH1, Note 7 } \\
\text { H-14-100952, SH1, Note 7 } \\
\text { H-14-100953, SH1, Note 7 }\end{array}$ & Field & CWP & $\begin{array}{l}\text { Prior to Cover Block } \\
\text { installation }\end{array}$ & QC-3 \\
\hline 1.4.C.A.C. 48 & $\begin{array}{l}\text { Final Special Protective } \\
\text { Coating inspection of pit \& } \\
\text { cover block }\end{array}$ & $\begin{array}{l}\text { W-314 Project Management } \\
\text { requirement }\end{array}$ & Field & CWP & $\begin{array}{l}\text { Prior to installation of } \\
\text { cover block }\end{array}$ & QC-3 \\
\hline 1.4.C.A.C. 49 & $\begin{array}{l}\text { Leak Detector/Drain Plug } \\
\text { Mock-up }\end{array}$ & $\begin{array}{l}\text { PDS-001, 3.7.2.1.1.5, } \\
\text { PDS-003, 3.2.1.1.4, 3.2.1.1.5, } \\
3.2 .1 .1 .6,3.2 .1 .2 .1,3.2 .1 .2 .2 \\
3.2 .1 .2 .3,3.2 .1 .3 .1,3.2 .1 .3 .2 \\
\text { 3.2.1.3.3, 3.2.2.2, } 3.2 .4 .3 \\
3.3 .2 .1\end{array}$ & & & $\begin{array}{l}\text { Mockup testing must be } \\
\text { completed and results } \\
\text { approved prior to } \\
\text { installation in the field. }\end{array}$ & $\begin{array}{l}\text { QC-3 } \\
\text { Design Agent- } 1,2,83 \\
\text { COG }-1,2,83 \\
\text { OPS }-1,2, \& 3\end{array}$ \\
\hline 1.4.C.A.C. 50 & $\begin{array}{l}\text { PLC Cabinet electrical and } \\
\text { instrumentation } \\
\text { configuration and code } \\
\text { compliance }\end{array}$ & $\begin{array}{l}\text { 1.4.C.A.C.39, Terminal Box } \\
\text { Conduit \& Cable Installation } \\
\text { Electrical \& Instrumentation } \\
\text { Configuration \& Code } \\
\text { Compliance }\end{array}$ & Field & CWP & Prior to ATP & NEC $\ln s p-3$ \\
\hline 1.4.C.A.C.51 & $\begin{array}{l}\text { PLC Cabinet wiring } \\
\text { (External) continuity }\end{array}$ & $\begin{array}{l}\text { 1.4.C.A.C.17, Leak Detector } \\
\text { Cabinet Installation Wiring } \\
\text { Continuity }\end{array}$ & Field & CWP & Complete prior to ATP & QC-3 \\
\hline 1.4.C.A.C.52 & $\begin{array}{l}\text { PLC Cabinet shielding } \\
\text { resistance to ground }\end{array}$ & $\begin{array}{l}\text { 1.4.C.A.C.23, Terminal Box } \\
\text { Conduit \& Cable Shielding } \\
\text { Resistance to Ground }\end{array}$ & Field & CWP & Complete prior to ATP & QC-3 \\
\hline 1.4.C.A.C.53 & $\begin{array}{l}\text { PLC Cabinet voltage } \\
\text { verification }\end{array}$ & $\begin{array}{l}\text { 1.4.C.A.C. } 27 \text {, Leak Detector } \\
\text { Installation Voltage Verification }\end{array}$ & Field & CWP & $\begin{array}{l}\text { Complete prior to } \\
\text { Energizing }\end{array}$ & QC-3 \\
\hline 4.6 .9 .658 & 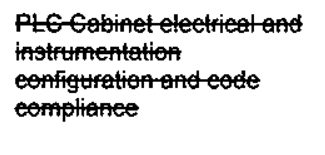 & 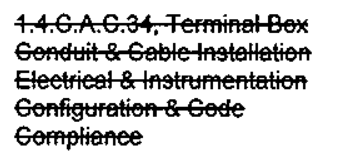 & Finter & GWF & PriotoATfP & AEG InOP-3 \\
\hline 1.4.C.A.C.54 & $\begin{array}{l}\text { Concrete Embedment } \\
\text { Installation }\end{array}$ & $\begin{array}{l}\text { W-314-C1, Rev 1, 03300, } \\
\text { Section } 3.4 .3\end{array}$ & Inspection & CWP & $\begin{array}{l}\text { Prior to Installing } \\
\text { Equipment }\end{array}$ & QC-2,3 \\
\hline 1.4.C.A.C.55 & $\begin{array}{l}\text { Leak Detection Relay } \\
\text { Cabinets }\end{array}$ & $\begin{array}{l}\text { W-314-C1, Rev 1, 13440, } \\
\text { Section 3.1.3 }\end{array}$ & Inspection & CWP & Prior to Installing Wiring & QC-2,3 \\
\hline 1.4.C.A.C.56 & $\begin{array}{l}\text { Intrinsically SafeWiring } \\
\text { Color Coded }\end{array}$ & $\begin{array}{l}\text { W-314-C1, Rev 1, 13440, } \\
\text { Section 3.1.4.1 }\end{array}$ & Field & CWP & Prior to ATP & QC-2,3 \\
\hline 1.4.C.A.C. 57 & Intrinsically Safe Wiring & $\begin{array}{l}\text { W-314-C1, Rev 1, 13440, } \\
\text { Section 3.1.4.2, 3.1.4.3, } \\
\text { 3.1.4.4, 3.1.4.5 }\end{array}$ & Field & CWP & Prior to ATP & $\mathrm{QC}-2,3$ \\
\hline 1.4.C.A.C.58 & Valves & $\begin{array}{l}\text { W-314-C1, Rev 1, 15493, } \\
\text { Section } 3.2 .5,3.2 .5 .1,3.2 .5 .2 \text {, } \\
\text { 3.2.5.3 }\end{array}$ & Field & CWP & 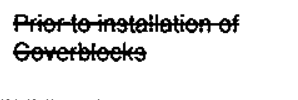 & QC-2,3 \\
\hline 1.4.C.A.C.59 & Labeling & $\begin{array}{l}\text { W-314-C1, Rev 1. } 16400 \text {, } \\
\text { Section 1.3.1b }\end{array}$ & Inspection & CWP & & QC-2.3 \\
\hline 1.4.C.A.C. 60 & Enclosure Type & $\begin{array}{l}\text { W-314-C1, Rev } 116400, \\
\text { Section } 2.2 .9\end{array}$ & Inspection & CWP & & QC-2,3 \\
\hline 4.4.6.A.6.64 & tobeling & $\begin{array}{l}\text { W-314G4,Rer 1, 1664, } \\
\text { Seetien } 1.3 .1 .2 b\end{array}$ & ingertion & GAP & & $06-2,3$ \\
\hline
\end{tabular}




\subsection{Acceptance Testing}

Acceptance testing will be conducted by utilizing ATPs. ATPs are separate "stand alone" documents which obtain their requirements from the applicable PDS, drawings, and vendor data. ATPS will ensure that the SSCs are fabricated and/or installed properly per the design. Detailed ATPS will be required after the CTI functional checks and/or inspections are completed and, will validate overall SSC installation as a whole. The acceptance criteria shall be specified in the test procedures. These tests will be performed on components in their installed condition but may not exercise the entire system as an operational unit. Testing of valve operators (with the valves connected), drain plug and leak detectors will be conducted with these components in their normal operating positions. Test conditions will meet the required code, design and authorization basis (i.e.. Basis for Operation. Technical Safety Requirements, and HNF-IP-1266, "TANK FARM OPERATIONS ADMINISTRATIVE CONTROLS") to verify that the system is functional and safe to operate. The table below describes the ATPS that will be performed for 241-AN-A Valve Pit modification and upgrade acceptance testing. An Acceptance Test Report (ATR) wi 11 be generated when a 11 test exceptions have been cleared and the test has been successfully completed. Hote that aceeptance-eriteria are-ineluded in the individual test doeument.

\section{TABLE 7-5}

\begin{tabular}{|c|c|c|c|c|c|}
\hline \multicolumn{6}{|c|}{ 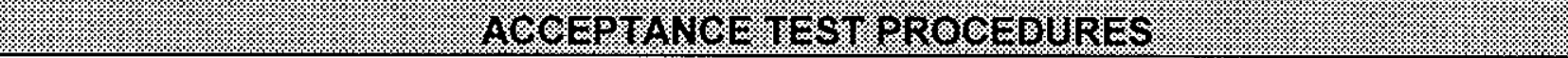 } \\
\hline 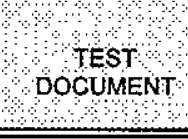 & $\begin{array}{l}\text { का } \\
\text { TEST DESCRIPTION } \\
\text { का }\end{array}$ & REFERENCE DOCUMENT & LOCATION & सNTERDEPENDENCIES & $\begin{array}{l}\text { 1) REVIEW } \\
\text { 2) APRROVAL, } \\
\text { 3) WITNESS } \\
\text { RESPONSIBLITIES }\end{array}$ \\
\hline $\begin{array}{l}\text { HNF- } \\
\text { ATP-314-003 }\end{array}$ & Pit Leak Detection & 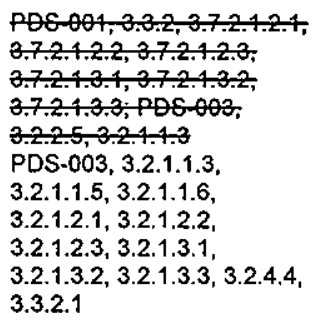 & Field & Leak Detector installed & $\begin{array}{l}\text { Design Agent -1,2 } \\
\text { Design Authority-1,2 } \\
\text { W-314 PM-1,2 } \\
\text { W-314 QA-1,2 } \\
\text { Chief Test Director-1 }\end{array}$ \\
\hline $\begin{array}{l}\text { HAF-26:33 } \\
\text { ATP-314-001 }\end{array}$ & $\begin{array}{l}\text { Valve Manifold and } \\
\text { Indicators (Position } \\
\text { Switches) }\end{array}$ & $\begin{array}{l}\text { PQS-002,3.3.2.3.7.2.1.1.4, } \\
3.7 .2 .2 .1,8.7 .2 .2 .3, \\
3.7 .2 .2 .4 \\
\text { PDS-002, 3.2.1.1.1, } \\
3.2 .2 .1 a, 3.2 .2 .1 \mathrm{~b}, 3.2 .2 .2\end{array}$ & Field & $\begin{array}{l}\text { After equipment } \\
\text { installation on cover block }\end{array}$ & Same as above. \\
\hline
\end{tabular}




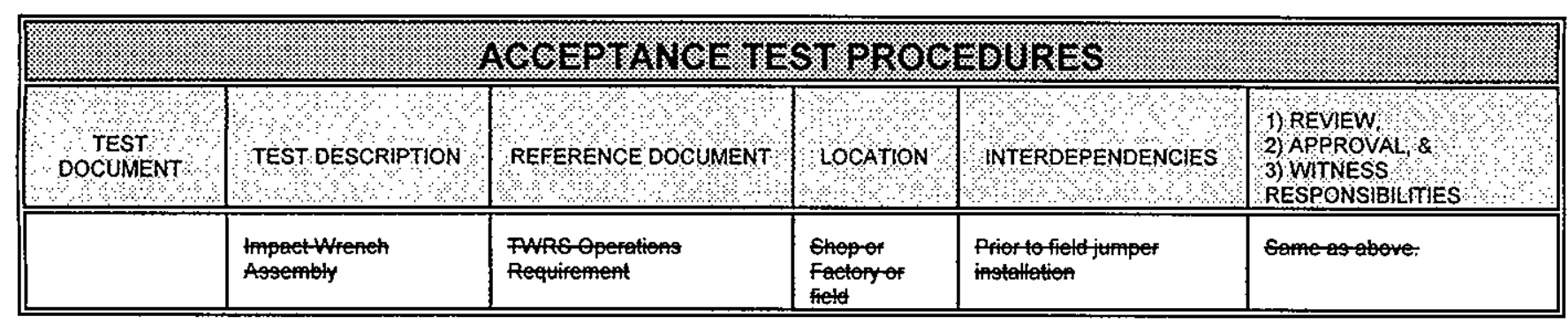

\subsection{Operational Testing}

Operational testing will be conducted by utilizing OTPS. OTPS will be planned, scheduled, and conducted, on the installed SSCs, after completion and acceptance of a 11 other test results. These detailed procedures shall determine if the installed SSCS meet the PDS functional requirements. Included in the OTP will be acceptance criteria to delineate pass/fail requirements. Operational testing is performed with actual plant equipment, operating procedures, and personnel. If an OTP has been generated but the testing of the corresponding SSC won't be performed for an extended period of time (e.g., a year or more), then the OTP must be reviewed again, prior to performance of the test, to ensure that it is still applicable. A Operational Test Report (OTR) will be generated when a11 test exceptions have been cleared and the test has been successfully completed. Testing of valve operators (with the valves connected), drain plug and leak detectors will be conducted with these components in their normal operating positions. Test conditions will meet the required code, design and authorization basis (i.e., Basis for Interim Operation. Technical Safety Requirements, and HNF-IP-1266. "TANK FARM OPERATIONS ADMINISTRATIVE CONTROLS") to verify that the system is functional and safe to operate. The following the desteribes the various seet of for-241-AN-A valve pit mifieations. Note that aceeptaneeriteria are ineluded in the individual test document. 
TABLE 7-6

\begin{tabular}{|c|c|c|c|c|c|c|}
\hline Test Section & 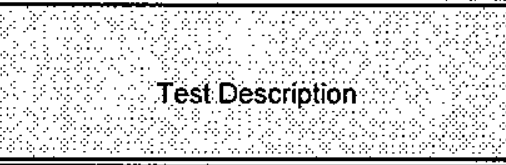 & $\begin{array}{lll}\text { Reference Document } \\
\text { Refold }\end{array}$ & Location & कocument & of prependencies & $\begin{array}{l}\text { 1) REVIEW } \\
\text { 2) APPROVAL \& } \\
\text { 3) WHNESS } \\
\text { RESPONSIBILITIES }\end{array}$ \\
\hline \multirow[t]{4}{*}{ OTP-314-XXX } & $\begin{array}{l}\text { The OTP will verify operations and } \\
\text { maintenance procedure adequacy. It will } \\
\text { verify valve cycling, actuator removal and } \\
\text { replacement, probe checks and } \\
\text { troubleshooting procedures. }\end{array}$ & $\begin{array}{l}\text { PDS-001, 3.3.3.1, } \\
3.3 .3 .2,3.3 .3 .3, \text { PDS- } \\
002,3.2 .1 .1 .1,3.2 .2 .1 \mathrm{a}, \\
3.2 .2 .1 \mathrm{~b}, 3.2 .2 .7 \\
3.3 .3 .1,3.7 .2 .2 .1, \text { POS- } \\
003,3.3 .3 .1,3.2 .2 .2\end{array}$ & Field & & $\begin{array}{l}\text { All equipment in } \\
\text { place. Locks \& tags } \\
\text { in place to prevent } \\
\text { inadvertent transfer of } \\
\text { waste. ATP-314-002, } \\
004,005 \text { complete }\end{array}$ & $\begin{array}{l}\text { Design Authority - 1,2 } \\
\text { Project Manager - 1,2 } \\
\text { Chief Test Director }-1,2 \\
\text { Startup Manager - 1,2 } \\
\text { QA }-1,2 \\
\text { Engineering Mgr - 1,2 }\end{array}$ \\
\hline & 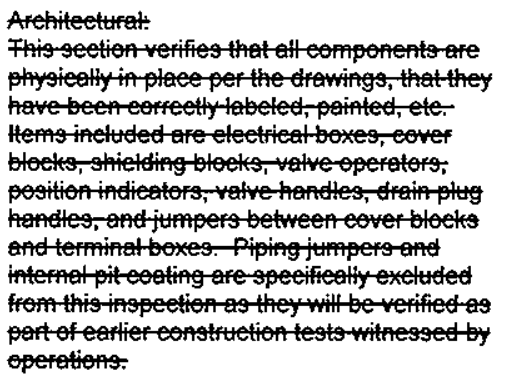 & 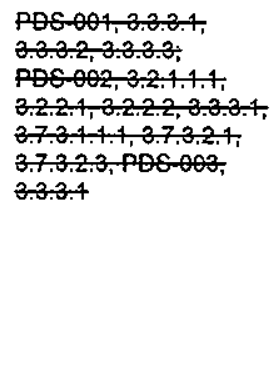 & Fieter & OTP-344-804 & $\begin{array}{l}\text { Allequipmentif } \\
\text { place, }\end{array}$ & 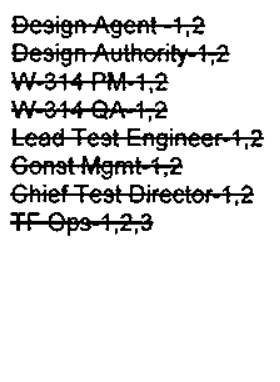 \\
\hline & 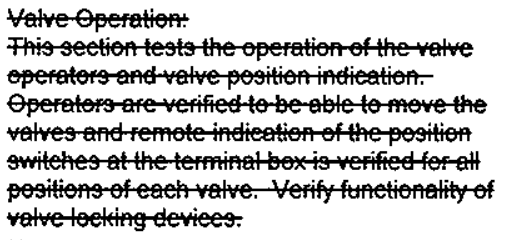 & & Fietet & OTP-314004 & 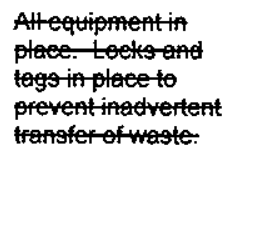 & Gameas abeve: \\
\hline & 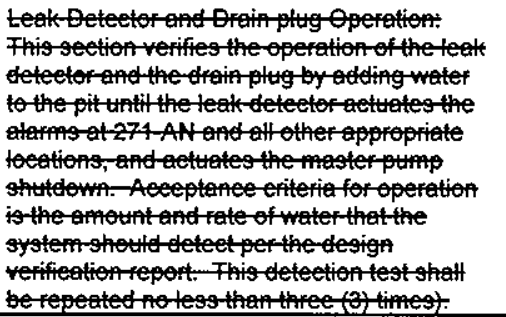 & PQS-003,3.2.4.4 & Field & OTP-344-004 & 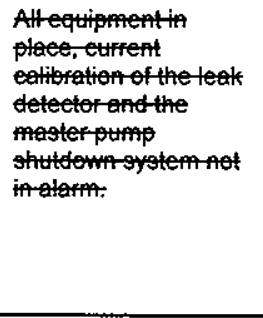 & Sameas ave. \\
\hline
\end{tabular}

\subsection{1-AN-A Valve Pit Test Completion}

241-AN-A Valve Pit testing will be considered successfully completed when the TEST COMPLETION CHECKLIST (attached to the end of this STEP) is completely signed off. This checklist is a "stand alone" document that wi 11 be listed on the 241-AN-A Valve Pit ABU (HNF-SD-W314-ABU-002) and included in the turnover of documents. This checklist will be completed by the $W-314$ Startup Manager or his designee. 


\section{REFERENCES}

HNF-PRO-229. Technical Procedure Standard. Fluor Daniel Hanford Company, Richland, Washington.

HWF-PRO-242. Rev. 1, Engineering Orawing Requirements, Fluor Daniel Hanford Company. Richland,

HNF-PRO-440, Engineering Document Change Control Requirements, Fluor Daniel Hanford Company, Richland, Washington.

HWF-PRO-446, Festing Practices Requirements, Fluor-Daniel Hanford Company, Richland, Washington.

HNF-PRO-572, Project Acceptance and Closeout, Fluor Daniel Hanford Company, Richland, Washington.

HNF-PRO-1819, PHMC Engineering Requirements, Fluor Daniel Hanford Company, Richland, Washington.

HNF-PRO-224. Document Control Program Standards, Fluor Daniel Hanford Company, Richland, Washington

W-314-C1, Construction Specification for Tank Farm Restoration and Safe Operations AN Valve Pit Upgrades, Fluor Daniel Northwest, Inc., Richland, Washington.

W-314-P1, Procurement Specification Ba77 Valves AN Valve Pit Upgrades, Fluor Daniel Northwest, Inc., Richland, Washington.

W-314-P2, Procurement Specification Impact Wrench AN Valve Pit Upgrades, Fluor Daniel Northwest. Inc., Richland, Washington.

HNF-SD-W314-PDS-001, Rev. 0, Project Development Specification - Transfer Piping. Fluor Daniel Northwest, Inc., Richland, Washington.

HNF-SD-W314-PDS-002, Rev. 0, Project Development Specification for Valve Pit Manifold, Fluor Daniel Northwest, Inc., Richland, Washington.

HNF-SD-W314-PDS-003, Rev. 0, Project Development Specification for Pit Leak Detection, Fluor Daniel Northwest, Inc., Richland, Washington.

HNF-SD-W314-PDS-005, Rev. 0, Project Development Specification for Special Protective Coating. Fluor Daniel Northwest, Inc., Richland, Washington.

HNF-1534, Rev. 0, Requirements Verification Report for AN Valve Pit upgrades (WBS 1.4.C), 
Fluor Daniel Northwest, Inc., Richland, Washington.

HNF-SD-W314-ABU-002. Rev. 0. AN-A VALVE PIT UPGRADE Acceptance For Beneficial Use, Rev. 0 , Numatec Hanford Corporation, Richland, Washington.

HNF-SD-W314-TEP-001, Rev. 0, Test and Evaluation Plan for W-314 Tank Farm Restoration and Safe Operations, Numatec Hanford Corporation, Richland, Washington.

HNF-CM-3-5, Document Control and Records Management, Fluor Daniel Hanford Company, Richland, Washington.

HNF-IP-0842, TWRS Administration, Fluor Daniel Hanford Company, Richland, Washington.

HNF-IP-1266, Tank Farm Operations Administrative Controls, Fluor Daniel Hanford Company, Richland, Washington.

HNF-SD-WM-BI0-001, Rev. 1. TWRS Basis for Interim Operation, Fluor Daniel Hanford Corporation, Richland, Washington.

W-314-C1-1, Rev. B, Acceptance Inspection Plan. Fluor Daniel Hanford Corporation. Richland. Washington. 
HNF-1733, Rev. 4

241-AN-A VALVE PIT UPGRADE TEST COMPLETION CHECKLIST

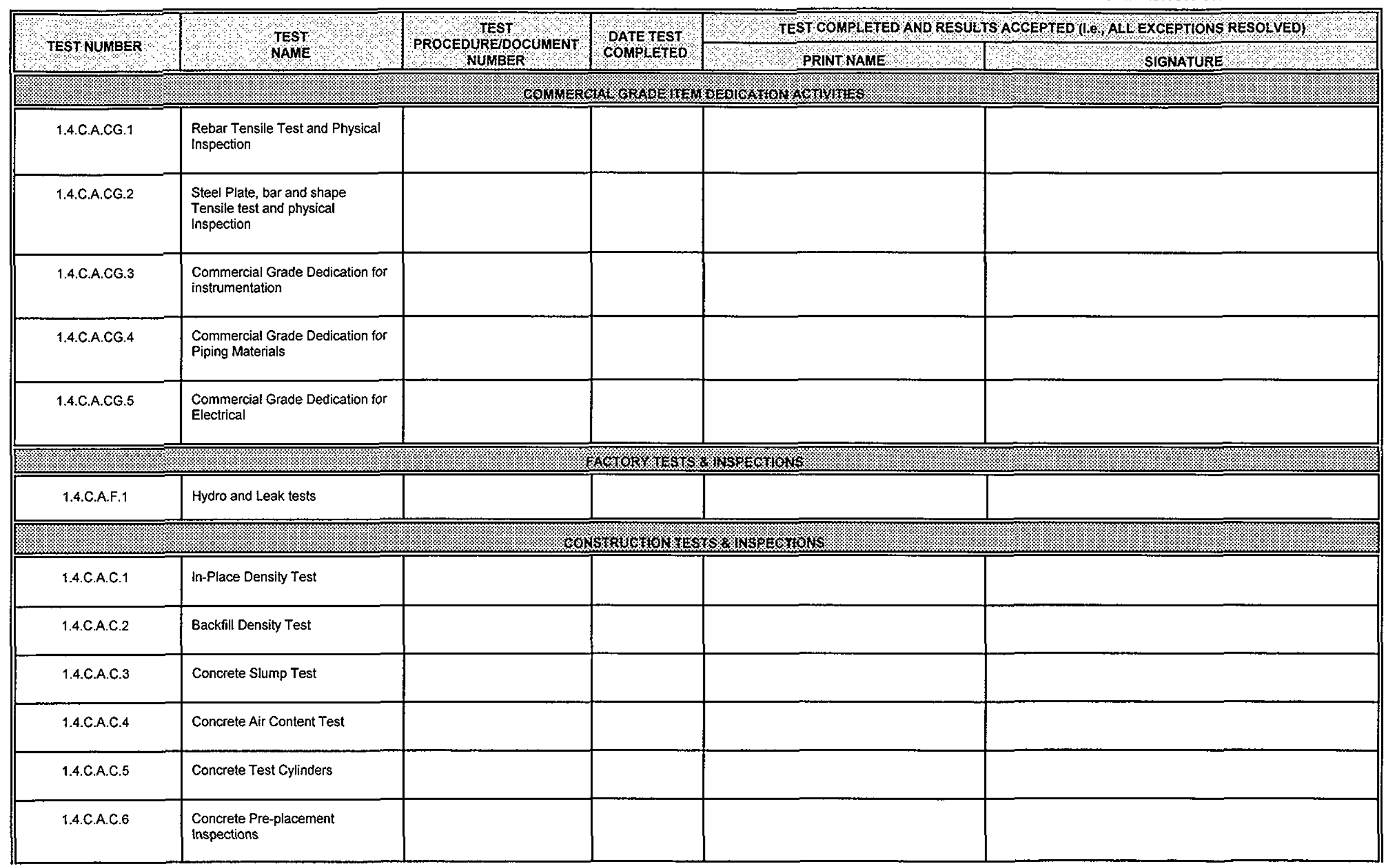


HNF-1733, Rev. 4

241-AN-A VALVE PIT UPGRADE TEST COMPLETION CHECKLIST

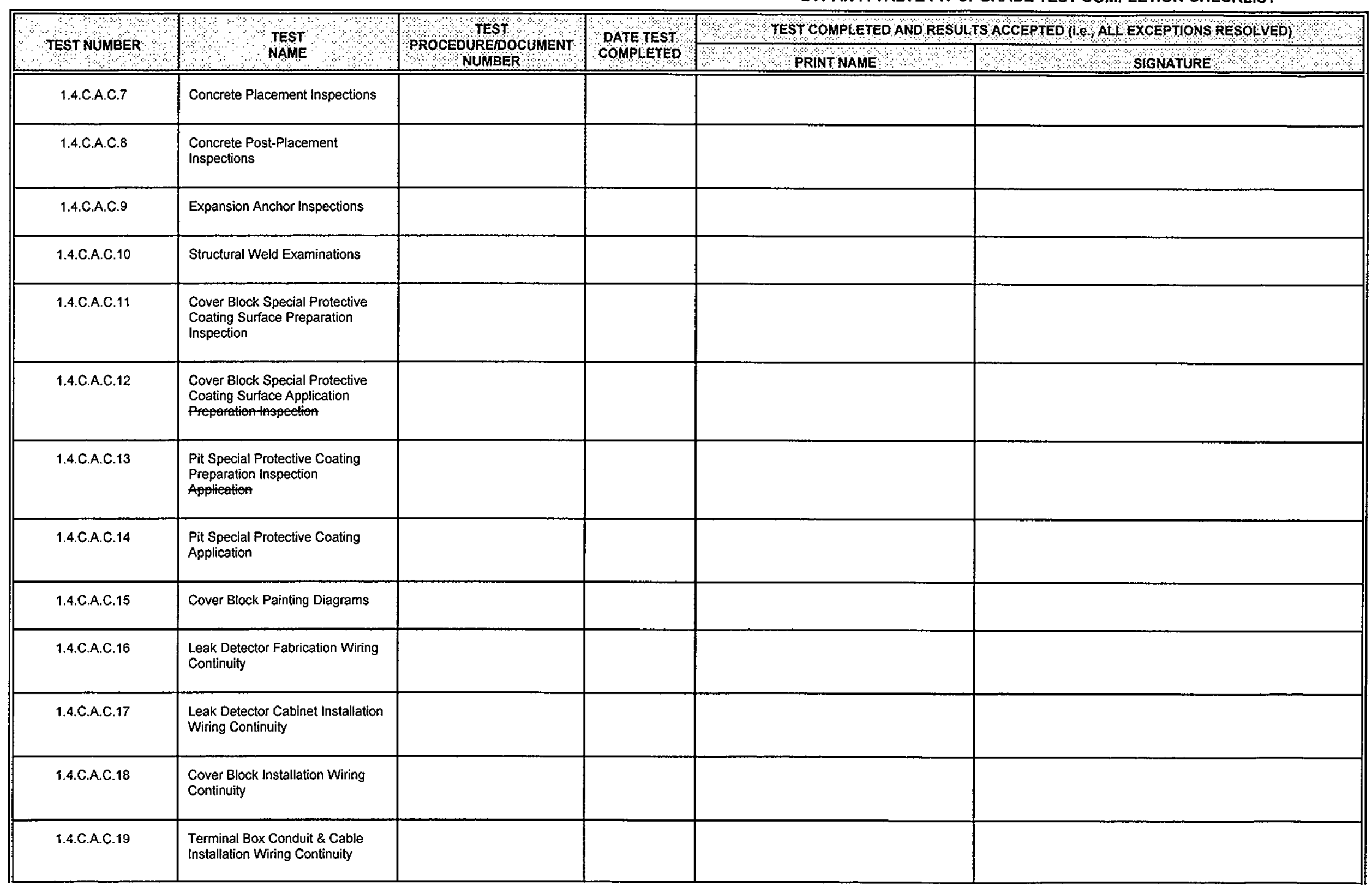


HNF-1733, Rev. 4

241-AN-A VALVE PIT UPGRADE TEST COMPLETION CHECKLIST

\begin{tabular}{|c|c|c|c|c|c|}
\hline \multirow{2}{*}{ TEST NUMBER } & \multirow{2}{*}{ TEST } & \multirow{2}{*}{ PROCEDUREDDOCUMENT } & \multirow{2}{*}{$\begin{array}{l}\text { DATETEST } \\
\text { COMPLETED }\end{array}$} & \multicolumn{2}{|c|}{ TEST COMPLETED AND RESULTS ACCEPTEO (L \& ALL EXCEPTIONS RESOLVED) } \\
\hline & & & & PRINTNAME & SIGNATURE \\
\hline 1.4.C.A.C.20 & $\begin{array}{l}\text { Valve Limit Switch Wiring } \\
\text { Continuity }\end{array}$ & & & & \\
\hline 1.4.C.A.C. 21 & $\begin{array}{l}\text { Leak Detector Installation Wiring } \\
\text { Continuity }\end{array}$ & & & & \\
\hline 1.4.C.A.C.22 & $\begin{array}{l}\text { Cover Block Wiring Shielding } \\
\text { Resistance to Ground }\end{array}$ & & & & \\
\hline 1.4.C.A.C.23 & $\begin{array}{l}\text { Terminal Box Conduit \& Cable } \\
\text { Shielding Resistance to Ground }\end{array}$ & & & & \\
\hline 1.4.C.A.C. 24 & $\begin{array}{l}\text { Valve Limit Switch Shielding } \\
\text { Resistance to Ground }\end{array}$ & & & & \\
\hline 1.4.C.A.C.25 & $\begin{array}{l}\text { Leak Detector Shielding } \\
\text { Resistance to Ground }\end{array}$ & & & & \\
\hline 1.4.C.A.C.26 & $\begin{array}{l}\text { Leak Detector Fabrication } \\
\text { Voitage Verification }\end{array}$ & & & & \\
\hline 1.4.C.A.C. 27 & $\begin{array}{l}\text { Leak Detector Installation Voltage } \\
\text { Verification }\end{array}$ & & & & \\
\hline 1.4.C.A.C.28 & $\begin{array}{l}\text { Holiday Testing of Piping } \\
\text { Protective Coatings }\end{array}$ & & & & \\
\hline 1.4.C.A.C. 29 & $\begin{array}{l}\text { Piping Fabrication Welding } \\
\text { Non-Destructive Examination }\end{array}$ & & & & \\
\hline 1.4.C.A.C. 30 & $\begin{array}{l}\text { Jumper Fabrication Welding } \\
\text { Non-Destructive Examination }\end{array}$ & & & & \\
\hline 1.4.C.A.C.31 & $\begin{array}{l}\text { Piping Fabrication Cleanliness } \\
\text { Inspection }\end{array}$ & & & & \\
\hline 1.4.C.A.C.32 & $\begin{array}{l}\text { Piping Fabrication Pressure } \\
\text { Tests }\end{array}$ & & & & \\
\hline
\end{tabular}


HNF-1733, Rev. 4

241-AN-A VALVE PIT UPGRADE TEST COMPLETION CHECKLIST

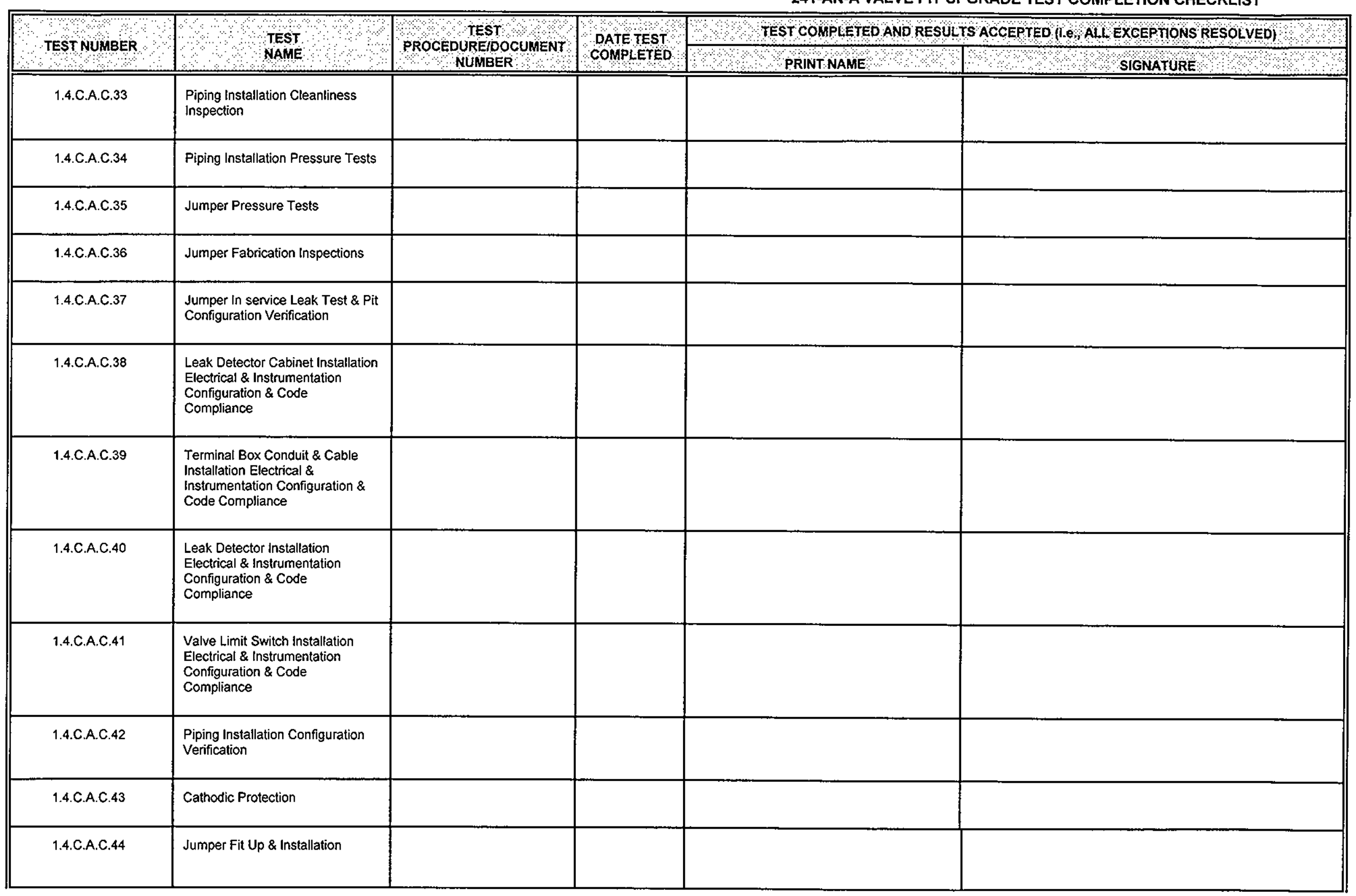


HNF-1733, Rev. 4

241-AN-A VALVE PIT UPGRADE TEST COMPLETION CHECKLIST

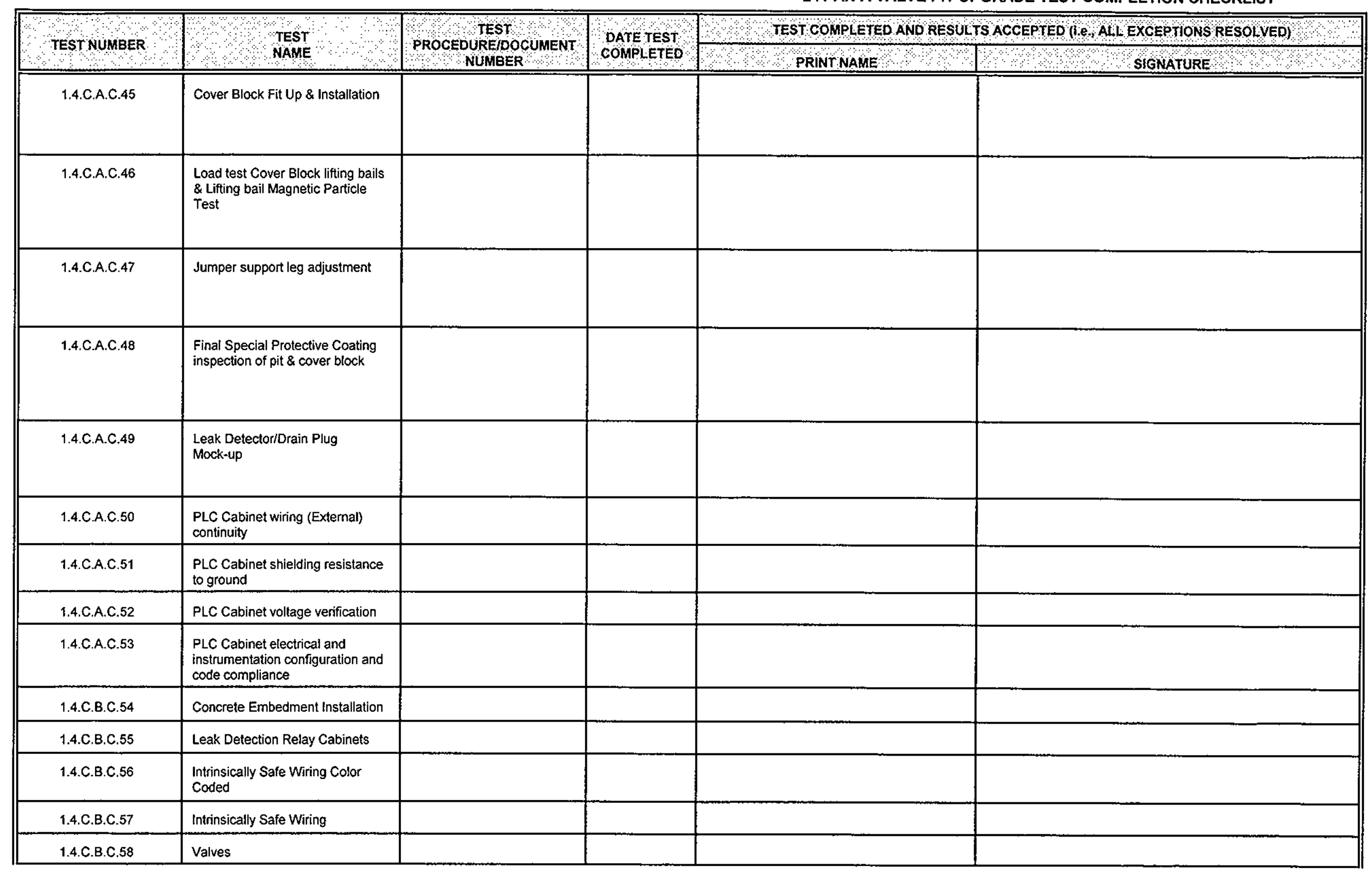


HNF-1733, Rev. 4

241-AN-A VALVE PIT UPGRADE TEST COMPLETION CHECKLIST

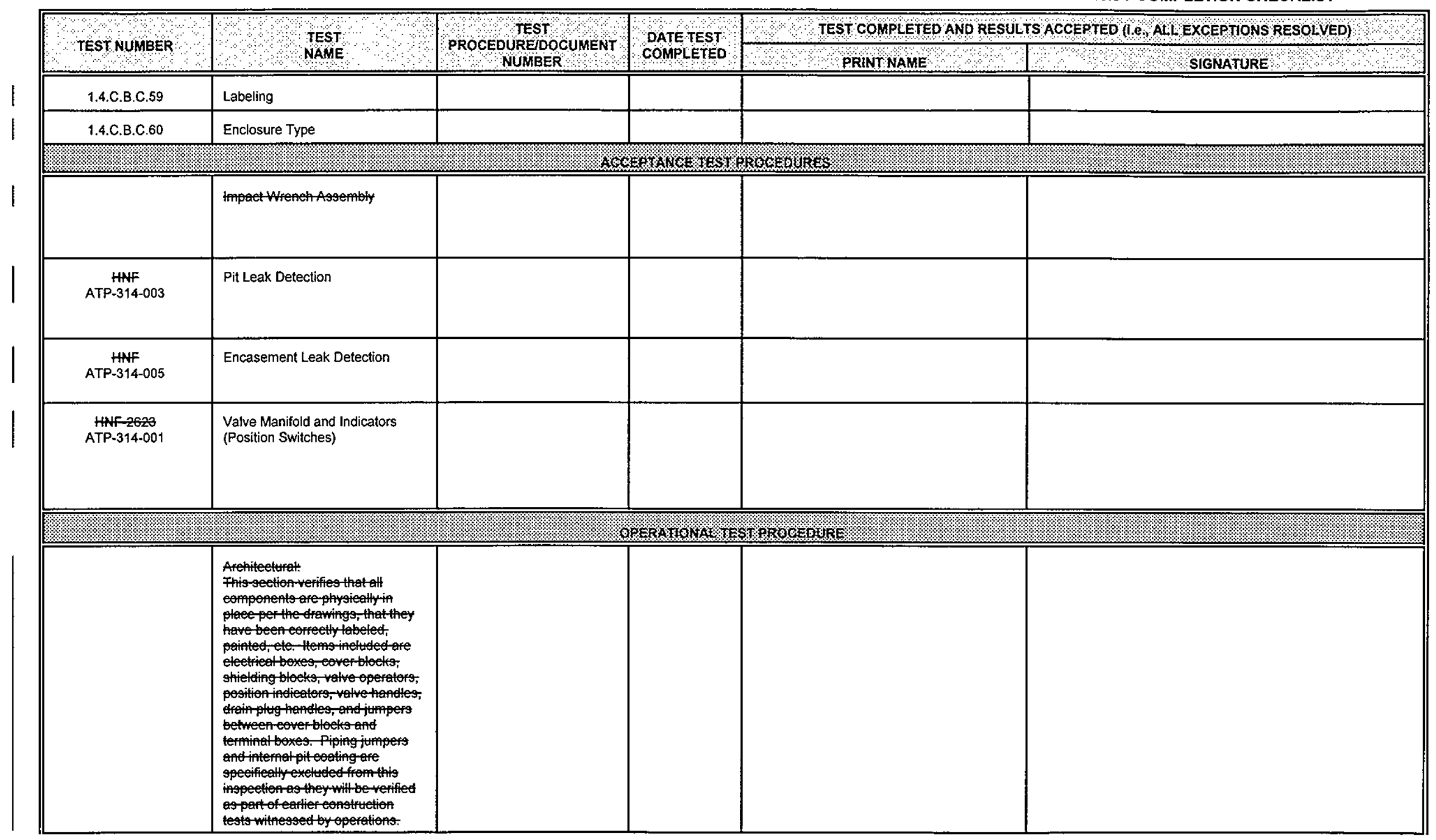




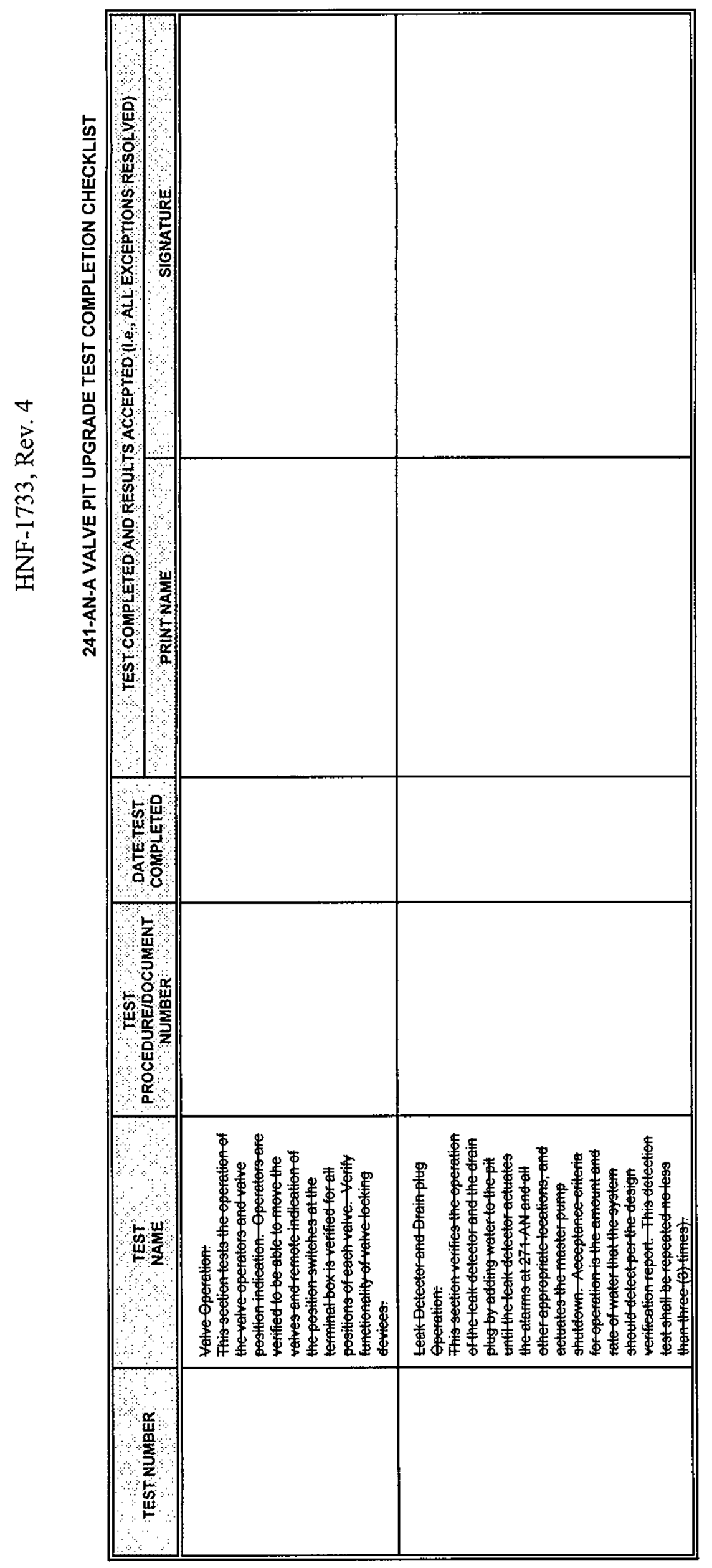

กิ 


\section{HNF-1733, REV. 4}

241-AN-A VALVE PIT LOGIC DIAGRAM

(SEE TEP FIGURE B-3 FOR HIGHER LEVEL LOGIC)

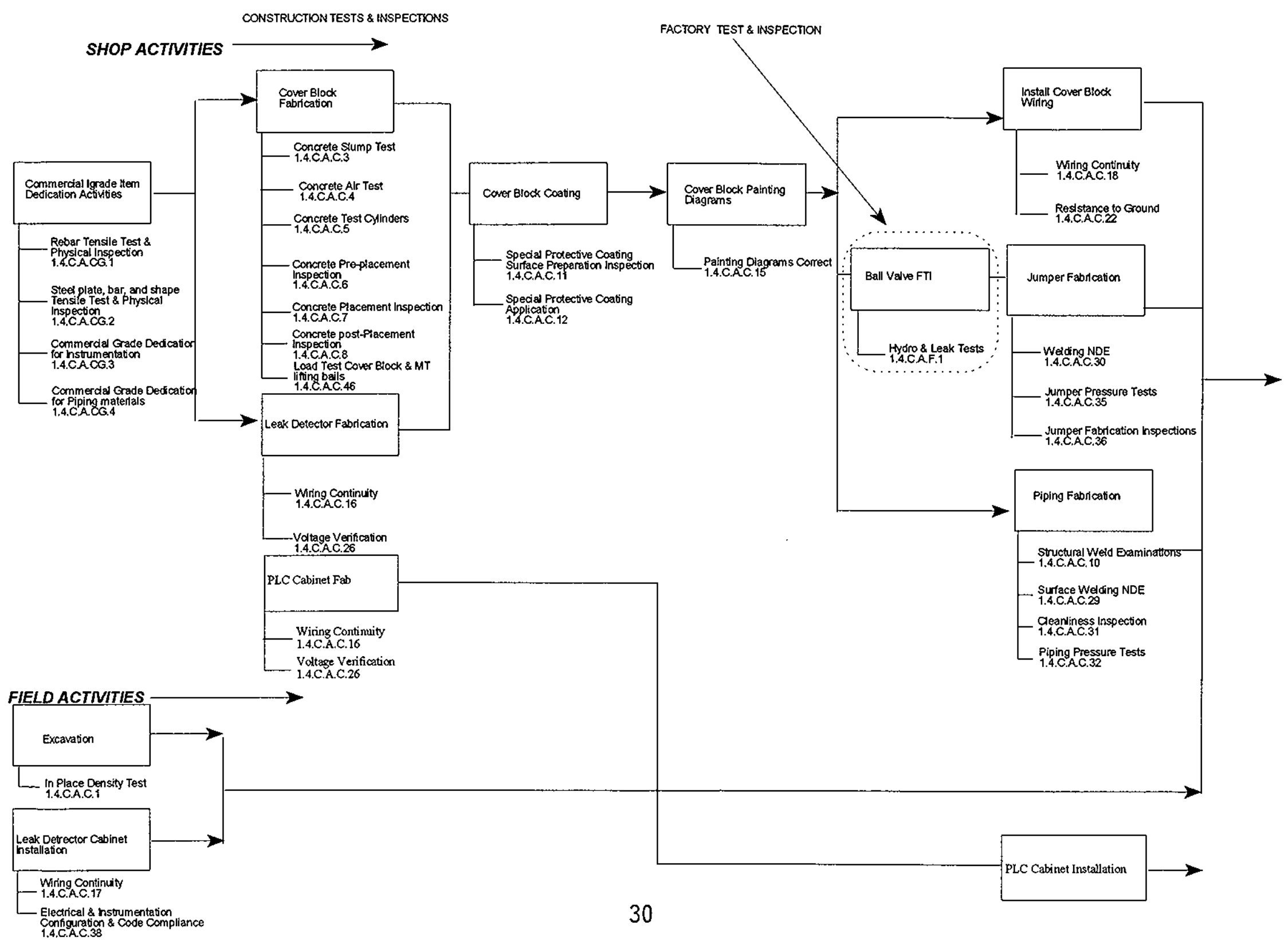




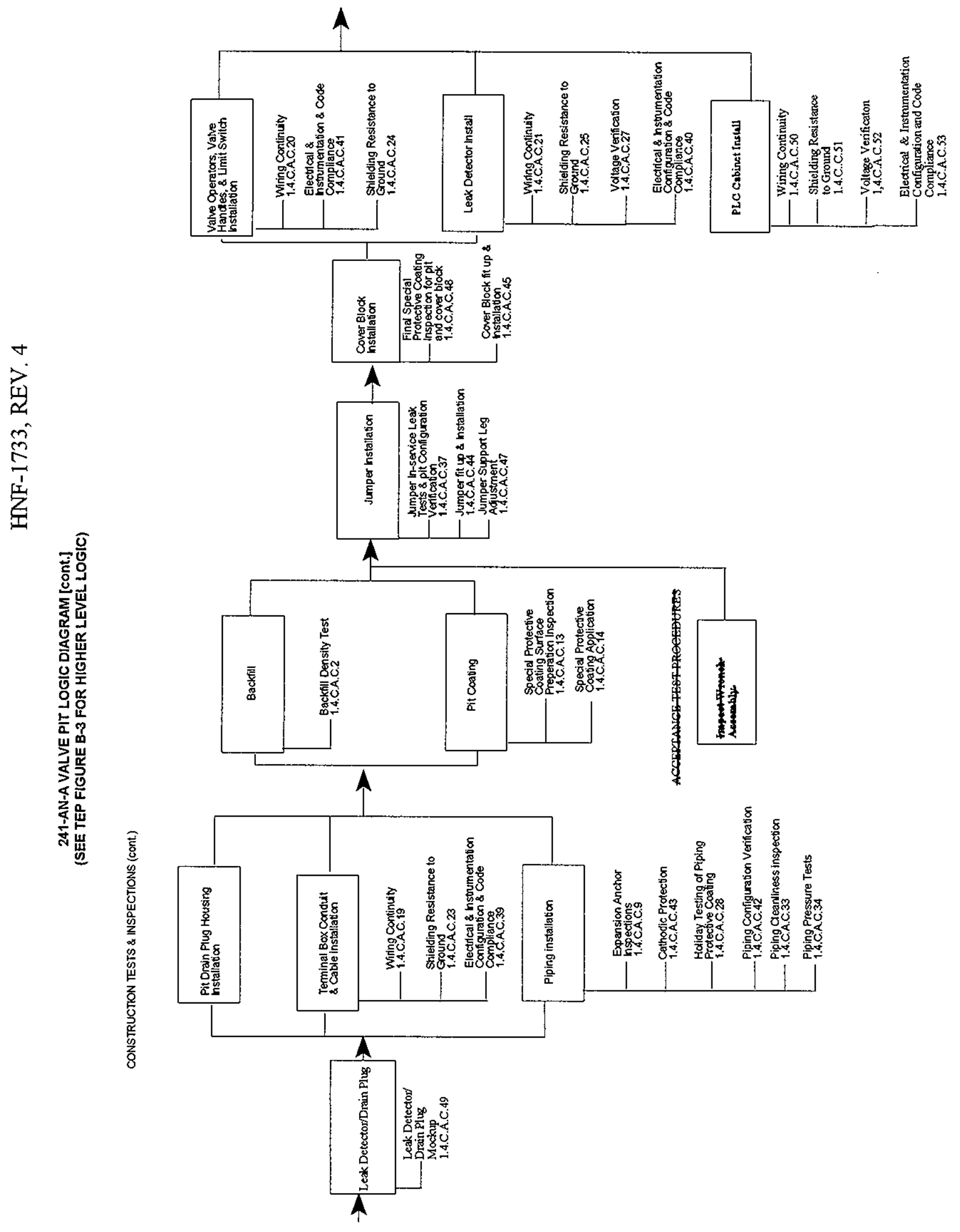

